\title{
Iterative Algorithms for a Generalized System of Mixed Variational-Like Inclusion Problems and Altering Points Problem
}

\author{
Monairah Alansari ${ }^{1}$, Mohd. Akram ${ }^{2,3, *}$, Mohd. Dilshad ${ }^{4}$ \\ ${ }^{1}$ Department of Mathematics, Faculty of Science, King Abdulaziz University, Jeddah, KSA \\ ${ }^{2}$ Department of Mathematics, Faculty of Science, Islamic University of Madinah, Madinah, KSA \\ ${ }^{3}$ Department of Mathematics, Aligarh Muslim University, Aligarh, India \\ ${ }^{4}$ Department of Mathematics, Faculty of Science, University of Tabuk, Tabuk, KSA
}

\begin{abstract}
In this article, we introduce and study a generalized system of mixed variational-like inclusion problems involving $\alpha \beta$-symmetric $\eta$-monotone mappings. We use the resolvent operator technique to calculate the approximate common solution of the generalized system of variational-like inclusion problems involving $\alpha \beta$-symmetric $\eta$-monotone mappings and a fixed point problem for nonlinear Lipchitz mappings. We study strong convergence analysis of the sequences generated by proposed Mann type iterative algorithms. Moreover, we consider an altering points problem associated with a generalized system of variational-like inclusion problems. To calculate the approximate solution of our system, we proposed a parallel $S$-iterative algorithm and study the convergence analysis of the sequences generated by proposed parallel $S$-iterative algorithms by using the technique of altering points problem. The results presented in this paper may be viewed as generalizations and refinements of the results existing in the literature.
\end{abstract}

Keywords Resolvent operator, $\alpha \beta$-symmetric $\eta$-monotone mapping, Altering points, Parallel $S$-iterative algorithm, Lipschiz mapping.

AMS 2010 subject classifications 49J40, 47J25, 47H10

DOI: $10.19139 /$ soic-2310-5070-884

\section{Introduction}

The theory of variational inequality was introduced by Hartmann and Stampacchia [17] in 1966 as a tool to study partial differential equations with applications. It has emerged as a powerful tool for wide class of unrelated problems arises in various branches of physical, engineering, pure and applied sciences in a unified and general framework, see; for example, [3, 4, 5, 8, 9, 10, 21]. Variational inequalities have been extended and generalized in different directions by using novel and innovative techniques for their own sake as well as for their applications. In 1989, Parida et al. [26] studied a generalized form of variational inequalities. They called it variational-like inequality problem and established its relationship with mathematical programming problem. Variational-like inequality problem has many important and novel applications in economics and optimizations, see; for example, $[7,32,35]$.

There are a substantial number of numerical methods including projection methods, Wiener-Hopf equations techniques, auxiliary principle technique, descent, and Newton methods, see; for example [11, 12, 13, 14, 24, 25] to solve variational and variational-like inequalities. Projection method and its variant forms represent important

\footnotetext{
${ }^{*}$ Correspondence to: Mohd. Akram (Email: akramkhan_20@redidiffmail.com). Department of Mathematics, Faculty of Science, Islamic University of Madinah, Madinah, KSA.
}

ISSN 2310-5070 (online) ISSN 2311-004X (print)

Copyright (C) 2020 International Academic Press 
tools for finding the approximate solutions of various type of variational and variational-like inequalities. It is worth mentioning that it is not possible to find the projection of the solution except in very simple cases. In addition, there are classes of variational inequalities, see; for example, [12, 13, 24, 25] for which the projection type methods cannot be applied to study the existence of the solution and to compute the approximate solution. These facts motivated us to use resolvent operator and altering points techniques to solve them efficiently. By using resolvent operators one may develop powerful and efficient iterative algorithms for several classes of variational inequalities. In fact, proximal algorithms are well suited to the problems of substantial recent interests involving large or higher dimensional data sets.

In this paper, we consider a generalized system of mixed variational-like inclusion problems involving $\alpha \beta$ symmetric $\eta$-monotone mapping and a fixed point problem of nonlinear Lipschitz mapping in Hilbert spaces. We use resolvent operator technique to calculate approximate common solution of generalized system of mixed variational-like inclusion problems involving $\alpha \beta$-symmetric $\eta$-monotone mapping and a fixed point problem for nonlinear Lipchitz mappings. We discuss the strong convergence analysis of the sequences generated by proposed Mann type iterative algorithm under some mild conditions. Moreover, we consider an altering points problem associated to generalized system of variational-like inclusion problems and propose a parallel $S$-iterative algorithm. We establish the existence of solution for our considered system of variational inclusions by using the technique of altering points problem. Finally, we prove convergence result for the proposed parallel $S$-iterative algorithm. The results presented in this paper improve and extend the results existing in the literature, see; for example, $[1,6,18,19,27,31,33,36]$.

Let $\mathcal{H}$ be a real Hilbert space equipped with inner product $\langle\cdot, \cdot\rangle$ which induces norm $\|\cdot\|$ and $d$ be the metric induced by the norm $\|\cdot\|$. Let $K$ be a nonempty closed and convex subset of $\mathcal{H}, \mathcal{C B}(\mathcal{H})$ be the family of all nonempty closed and bounded subsets of $\mathcal{H}$ and let $\mathcal{D}(\cdot, \cdot)$ be the Hausdorff metric on $\mathcal{C B}(\mathcal{H})$ defined by

$$
\mathcal{D}(X, Y)=\max \left\{\sup _{x \in X} d(x, Y), \sup _{y \in Y} d(X, y)\right\}, \forall X, Y \in \mathcal{C B}(\mathcal{H})
$$

where $d(x, Y)=\inf _{y \in Y} d(x, y)$ and $d(X, y)=\inf _{x \in X} d(x, y)$.

Before presenting our main results, we collect relevant definitions and results which will be needed in our subsequent discussion.

Definition 1.1. A nonlinear mapping $T: \mathcal{H} \rightarrow \mathcal{H}$ is said to be Lipchitz continuous if there exists a constant $k>0$ such that

$$
\|T x-T y\| \leq k\|x-y\|, \forall x, y \in \mathcal{H} .
$$

If $k=1$, the mapping $T$ is called nonexpansive. We denote the set of all fixed points of $T$ by $F(T)$, that is, $F(T)=\{x \in \mathcal{H}: T x=x\}$.

Definition 1.2. Let $\eta: \mathcal{H} \times \mathcal{H} \rightarrow \mathcal{H} ; A, B: \mathcal{H} \rightarrow \mathcal{H}$ be the single-valued mappings and let $M: \mathcal{H} \times \mathcal{H} \rightarrow \mathcal{H}$ be a nonlinear mapping. Then

(i) $M(A, \cdot)$ is said to be $\alpha$-strongly $\eta$-monotone with respect to $A$, if there exists a constant $\alpha>0$ such that

$$
\langle M(A x, u)-M(A y, u), \eta(y, x)\rangle \geq \alpha\|x-y\|^{2}, \forall x, y, u \in \mathcal{H} ;
$$

(ii) $M(\cdot, B)$ is said to be $\beta$-relaxed $\eta$-monotone with respect to $B$, if there exists a constant $\beta>0$ such that

$$
\langle M(u, B x)-M(u, B y), \eta(y, x)\rangle \geq(-\beta)\|x-y\|^{2}, \forall x, y, u \in \mathcal{H} ;
$$

(iii) $M(A, B)$ is said to be $\alpha \beta$-symmetric $\eta$-monotone with respect to $A$ and $B$, if $M(A, \cdot)$ is $\alpha$-strongly $\eta$ monotone with respect to $A$ and $M(\cdot, B)$ is $\beta$-relaxed $\eta$-monotone with respect to $B$; 
(iv) $M(\cdot, \cdot)$ is said to be $\xi$-mixed Lipschitz continuous with respect to $A$ and $B$, if there exists a constant $\xi>0$ satisfying

$$
\|M(A x, B x)-M(A y, B y)\| \leq \xi\|x-y\|, \forall x, y \in \mathcal{H}
$$

(v) $\eta$ is said to be $\tau$-Lipschitz continuous, if there exists a constant $\tau>0$ such that

$$
\|\eta(x, y)\| \leq \tau\|x-y\|, \forall x, y \in \mathcal{H} .
$$

Definition 1.3. Let $A, B: \mathcal{H} \rightarrow \mathcal{H} ; M, N: \mathcal{H} \times \mathcal{H} \rightarrow \mathcal{H}$ be the single-valued mappings and $T, P: \mathcal{H} \rightarrow \mathcal{C B}(\mathcal{H})$ be the set-valued mappings. Then

(i) $T$ is said to be $\mathcal{D}$-Lipschitz continuous, if there exists a constant $\delta_{T}>0$ such that

$$
\mathcal{D}(T(x), T(y)) \leq \delta_{T}\|x-y\|, \forall x, y \in \mathcal{H} ;
$$

(ii) $M$ is said to be mixed strongly monotone with respect to $A$ and $B$, if there exists a constant $k>0$ such that

$$
\langle M(A x, B x)-M(A y, B y), x-y\rangle \geq k\|x-y\|^{2}, \forall x, y \in \mathcal{H} ;
$$

(iii) $P$ is said to be strongly monotone with respect to $N$ in the first argument, if there exists a constant $c>0$ such that

$$
\langle N(u, \cdot)-N(v, \cdot), x-y\rangle \geq c\|x-y\|^{2}, \forall x, y \in \mathcal{H} \text { and for some } u \in P(x), v \in P(y) ;
$$

(iv) $N$ is said to be $\gamma$-Lipschitz continuous in the first argument, if there exists a constant $\gamma>0$ such that

$$
\|N(u, \cdot)-N(v, \cdot)\| \leq \gamma\|u-v\|, \forall x \in \mathcal{H}, u \in P(x), v \in P(y) .
$$

Similarly, one can define strong monotonicity of $P$ in the second argument with respect to $N$ and Lipschitz continuity of $N$ in the second argument.

Let $\mathcal{H}$ be a real Hilbert space with norm $\|\cdot\|$. Define the norm $\|\cdot\|_{*}$ on $\mathcal{H} \times \mathcal{H}$ by

$$
\|(x, y)\|_{*}=\|x\|+\|y\|, \forall x, y \in \mathcal{H} .
$$

Note that $\left(\mathcal{H} \times \mathcal{H},\|\cdot\|_{*}\right)$ is a Banach space.

Definition 1.4. Let $A$ and $B$ be two nonempty subsets of a metric space $X$ and let $P: A \rightarrow B$ and $Q: B \rightarrow A$ be mappings. Then $x \in A$ and $y \in B$ are called altering points of the mappings $P$ and $Q$, if

$$
\left\{\begin{array}{l}
P(x)=y, \\
Q(y)=x .
\end{array}\right.
$$

We denote the set of altering points of the mappings $P: A \rightarrow B$ and $Q: B \rightarrow A$ by

$$
\operatorname{Alt}(P, Q)=\{(x, y) \in A \times B: P(x)=y \text { and } Q(y)=x\} .
$$

Example 1.1. Let $\mathcal{H}=\mathbb{R}$ and $C=D=[0, k], k>0$. Define $P, Q: \mathcal{H} \rightarrow \mathcal{H}$ by $P(x)=Q(x)=k-x$. Then $Q P: C \rightarrow C$ is defined by $Q P(x)=Q(k-x)=x$ and $P Q: D \rightarrow D$ is defined by $P Q(x)=P(k-x)=x$. It is easy to see that each point of $C$ is a fixed point of $Q P$ and each point of $D$ is a fixed point of $P Q$. Thus, altering points $x \in C$ and $y \in D$ are given by

$$
\text { Alt }(P, Q)=\{(x, y) \in C \times D: x+y=k\} .
$$

Example 1.2. Let $\mathcal{H}=\mathbb{R}, C=[0,2]$, and $D=[2,4]$. Define $P: C \rightarrow D$ by $P(x)=x+2, \forall x \in C$ and $Q: D \rightarrow$ $C$ by $Q(x)=\frac{x^{2}}{8}, \forall x \in D$. Note that, $P Q(x)=\frac{x^{2}}{8}+2, \forall x \in D$ and $Q P(x)=\frac{(x+2)^{2}}{8}, \forall x \in C$. Then $(2,4)$ are altering points of $P$ and $Q$, i.e., $(2,4) \in \operatorname{Alt}(P, Q)$. 
Definition 1.5. [38]. A functional $f: \mathcal{H} \times \mathcal{H} \rightarrow \mathbb{R} \cup\{+\infty\}$ is said to be 0-diagonally quasi-concave (in short, $0-D Q C V)$ in $x_{i}$, if for any finite set $\left\{x_{1}, x_{2}, \cdots, x_{n}\right\} \subset \mathcal{H}$ and for any $y=\sum_{i=1}^{n} \lambda_{i} x_{i}$ with $\lambda_{i} \geq 0$ and $\sum_{i=1}^{n} \lambda_{i}=1$,

$$
\min _{1 \leq i \leq n} f\left(x_{i}, y\right) \leq 0
$$

Definition 1.6. Let $\eta: \mathcal{H} \times \mathcal{H} \rightarrow \mathcal{H}$ be a single-valued mapping. A proper functional $\phi: \mathcal{H} \rightarrow \mathbb{R} \cup\{+\infty\}$ is said to be $\eta$-subdifferentiable at a point $x \in \mathcal{H}$, if there exists a point $f^{*} \in \mathcal{H}$ such that

$$
\phi(y)-\phi(x) \geq\left\langle f^{*}, \eta(y, x)\right\rangle, \forall y \in \mathcal{H} ;
$$

where $f^{*}$ is called $\eta$-subdgradient of $\phi$ at $x \in \operatorname{dom} \phi$. The set of all $\eta$-subgradients of $\phi$ at $x$ is denoted by $\partial_{\eta} \phi(x)$. The mapping $\partial_{\eta} \phi: \mathcal{H} \rightarrow 2^{\mathcal{H}}$ defined by

$$
\partial_{\eta} \phi(x)= \begin{cases}f^{*} \in \mathcal{H}: \phi(y)-\phi(x) \geq\left\langle f^{*}, \eta(y, x)\right\rangle, \forall y \in \mathcal{H}, & x \in \operatorname{dom} \phi \\ \emptyset, & x \notin \operatorname{dom} \phi\end{cases}
$$

is said to be $\eta$-subdifferential of $\phi$ at $x$.

Definition 1.7. Let $\eta: \mathcal{H} \times \mathcal{H} \rightarrow \mathcal{H} ; A, B: \mathcal{H} \rightarrow \mathcal{H}$ be the single-valued mappings. Let $\phi: \mathcal{H} \rightarrow \mathbb{R} \cup\{+\infty\}$ be a proper, lower semicontinuous and $\eta$-subdifferentiable (may not be convex) functional and let $M: \mathcal{H} \times \mathcal{H} \rightarrow \mathcal{H}$ be a nonlinear mapping. If for any given point $z \in \mathcal{H}$ and $\rho>0$, there exists a unique point $x \in \mathcal{H}$ satisfying

$$
\langle M(A x, B x)-z, \eta(y, x)\rangle+\rho \phi(y)-\rho \phi(x) \geq 0, \forall y \in \mathcal{H},
$$

then the mapping $z \mapsto x$, denoted by $R_{\rho, M(\cdot, \cdot)}^{\partial_{\eta} \phi}(z)$ is called resolvent operator of $\phi$. Then, we have $z-$ $M(A x, B x) \in \rho \partial_{\eta} \phi(x)$ and it follows that $R_{\rho, M(\cdot, \cdot)}^{\partial_{\eta} \phi}(z)=\left[M(A, B)+\rho \partial_{\eta} \phi\right]^{-1}(z)$.

Lemma 1.1. [20]. Let $\mathcal{H}$ be a real Hilbert space. Let $\eta: \mathcal{H} \times \mathcal{H} \rightarrow \mathcal{H}$ be a continuous mapping such that $\eta(x, y)+\eta(y, x)=0, \forall x, y \in \mathcal{H}$; let $M: \mathcal{H} \times \mathcal{H} \rightarrow \mathcal{H}$ be an $\alpha \beta$-symmetric $\eta$-monotone mapping with respect to $A$ and $B$; let for any $z \in \mathcal{H}$, the function $h(y, x)=\langle z-M(A x, B x), \eta(y, x)\rangle$ be $0-D Q C V$ in $y$ and let $\phi: \mathcal{H} \rightarrow \mathbb{R} \cup\{+\infty\}$ be a proper, lower semicontinuous and $\eta$-subdifferentiable (may not be convex) functional. Then for any given constant $\rho>0$ and $z \in \mathcal{H}$, there exists a unique $x \in \mathcal{H}$ such that

$$
\langle M(A x, B x)-z, \eta(y, x)\rangle \geq \rho \phi(x)-\rho \phi(y), \forall y \in \mathcal{H},
$$

that is, $x=R_{\rho, M(\cdot, \cdot)}^{\partial_{\eta} \phi}(z)$.

Lemma 1.2. [20]. Let $\eta: \mathcal{H} \times \mathcal{H} \rightarrow \mathcal{H}$ be $\tau$-Lipschitz continuous such that $\eta(x, y)+\eta(y, x)=0, \forall x, y \in \mathcal{H}$; let $M: \mathcal{H} \times \mathcal{H} \rightarrow \mathcal{H}$ be an $\alpha \beta$-symmetric $\eta$-monotone mapping with respect to $A$ and $B$; let for any $z \in \mathcal{H}$, the function $h(y, x)=\langle z-M(A x, B x), \eta(y, x)\rangle$ be $0-D Q C V$ in $y$ and let $\phi: \mathcal{H} \rightarrow \mathbb{R} \cup\{+\infty\}$ be a proper, lower semicontinuous and $\eta$-subdifferentiable functional and let $\rho>0$ be any given constant. Then the resolvent operator $R_{\rho, M(\cdot, \cdot)}^{\partial_{\eta} \phi}$ of $\phi$ is $\frac{\tau}{\alpha-\beta}$-Lipschitz continuous, that is,

$$
\left\|R_{\rho, M(\cdot, \cdot)}^{\partial_{\eta} \phi}\left(z_{1}\right)-R_{\rho, M(\cdot, \cdot)}^{\partial_{\eta} \phi}\left(z_{2}\right)\right\| \leq \frac{\tau}{\alpha-\beta}\left\|z_{1}-z_{2}\right\|, \forall z_{1}, z_{2} \in \mathcal{H} .
$$

Example 1.3. Let $\mathcal{H}=\mathbb{R}, \eta: \mathcal{H} \times \mathcal{H} \rightarrow \mathcal{H}$ be a mapping defined by $\eta(y, x)=\frac{x-y}{c}, \forall x, y \in \mathcal{H}, c>0$ be any real number. Then, it is easy to verify that

(i) $\eta(x, y)+\eta(y, x)=0, \forall x, y \in \mathbb{R}$;

(ii) $|\eta(x, y)| \leq \frac{1}{c}|x-y|, \forall x, y \in \mathbb{R}$; i.e., $\eta$ is $\frac{1}{c}$-Lipschitz continuous. 
Let $M: \mathcal{H} \times \mathcal{H} \rightarrow \mathcal{H}$ and $A, B: \mathcal{H} \rightarrow \mathcal{H}$ be the mappings defined by $M(x, y)=x-y, A(x)=3 k x, B(x)=2 k x$ for any $x \in \mathbb{R}$ and $k>0$. Then

$$
\begin{aligned}
\langle M(A x, u)-M(A y, u), \eta(y, x)\rangle & =\langle A(x)-A(y), \eta(y, x)\rangle \\
& =\left\langle 3 k(x-y), \frac{x-y}{c}\right\rangle \\
& \geq \frac{3 k}{c}|x-y|^{2}, \forall x, y \in \mathbb{R}
\end{aligned}
$$

i.e., $M$ is $\frac{3 k}{c}$-strongly $\eta$-monotone with respect to $A$.

$$
\begin{aligned}
\langle M(u, B x)-M(u, B y), \eta(y, x)\rangle & =\langle-(B(x)-B(y)), \eta(y, x)\rangle \\
& =\left\langle-2 k(x-y), \frac{x-y}{c}\right\rangle \\
& \geq \frac{-2 k}{c}|x-y|^{2}, \forall x, y \in \mathbb{R}
\end{aligned}
$$

i.e., $M$ is $\frac{2 k}{c}$-relaxed $\eta$-monotone with respect to $B$. Hence, $M(A, B)$ is $\alpha \beta$-symmetric $\eta$-monotone mapping with respect to $A$ and $B$.

Next, for any $x, z \in \mathbb{R}$, the mapping

$$
\begin{aligned}
h(y, x) & =\langle z-M(A x, B x), \eta(y, x)\rangle \\
& =(z-k x)\left(\frac{x-y}{c}\right)
\end{aligned}
$$

is $0-D Q C V$ in $y$. If, it is false, then there exists a finite set $\left\{y_{1}, y_{2}, \cdots, y_{m}\right\}$ and $x_{0}=\sum_{i=1}^{m} t_{i} y_{i}$ with $t_{i} \geq 0$ and $\sum_{i=1}^{m} t_{i}=1$ such that for each $i=1,2, \cdots, m$,

$$
\begin{aligned}
0<h\left(y_{i}, x_{0}\right) & =\left\langle z-M\left(A x_{0}, B x_{0}\right), \eta\left(y_{i}, x_{0}\right)\right\rangle \\
& =\left(z-k x_{0}\right)\left(\frac{x_{0}-y_{i}}{m^{c}}\right) \\
& =\left(z-k x_{0}\right)\left(\frac{\sum_{i=1}^{m^{c}} t_{i} y_{i}-y_{i}}{c}\right)=0,
\end{aligned}
$$

which is not possible. Thus, for any $x, z \in \mathbb{R}$, the mapping $h(y, x)$ is is $0-D Q C V$ in $y$.

Lemma 1.3. Let $\left\{a_{n}\right\}$ and $\left\{b_{n}\right\}$ be two nonnegative real sequences satisfying the following conditions:

$$
a_{n+1} \leq\left(1-\lambda_{n}\right) a_{n}+b_{n}, \quad \forall n \geq n_{0}
$$

for some $n_{0} \in \mathbb{N},\left\{\lambda_{n}\right\} \subset(0,1)$ with $\sum_{n=0}^{\infty} \lambda_{n}=\infty, b_{n}=o\left(\lambda_{n}\right)$. Then $\lim _{n \rightarrow \infty} a_{n}=0$.

Lemma 1.4. Let $\left\{a_{n}\right\}$ and $\left\{b_{n}\right\}$ be two nonnegative real sequences satisfying the following inequality:

$$
a_{n+1} \leq k a_{n}+b_{n}, \forall n \in \mathbb{N}
$$

$k \in(0,1)$ with $\lim _{n \rightarrow \infty} b_{n}=0$. Then $\lim _{n \rightarrow \infty} a_{n}=0$.

\section{Formulation of the Problem and Existence Results}

Let $\mathcal{H}$ be a real Hilbert space and assume that $I=\{1,2\}$ is an index set; for each $i \in I$, let $M_{i}, N_{i}: \mathcal{H} \times \mathcal{H} \rightarrow$ $\mathcal{H} ; A_{i}, B_{i}, g_{i}: \mathcal{H} \rightarrow \mathcal{H}$ be the single-valued mappings and $P, Q: \mathcal{H} \rightarrow \mathcal{C B}(\mathcal{H})$ be the multi-valued mappings; 
$\eta_{i}: \mathcal{H} \times \mathcal{H} \rightarrow \mathcal{H}$ be a single-valued nonlinear mapping and $\phi_{i}: \mathcal{H} \times \mathcal{H} \rightarrow \mathbb{R} \cup\{+\infty\}$ be mapping such that for fixed $x, y \in \mathcal{H}, \phi_{1}\left(g_{1}(\cdot), x\right)$ and $\phi_{2}\left(g_{2}(\cdot), y\right)$ be lower semi-continuous, $\eta$-subdifferential, proper functionals on $\mathcal{H}$ satisfying $g_{1}(\mathcal{H}) \cap \operatorname{dom}\left(\partial_{\eta} \phi\left(g_{1}(\cdot), x\right)\right) \neq \emptyset$ and $g_{2}(\mathcal{H}) \cap \operatorname{dom}\left(\partial_{\eta} \phi\left(g_{2}(\cdot), y\right)\right) \neq \emptyset$. We consider the following generalized system of multi-valued mixed variational-like inclusion problems $(G S M V L I P)$ :

Find $(x, y, u, v)$ such that $x, y \in \mathcal{H}, v \in P(x), u \in Q(y)$ and

$$
\operatorname{GSMVLIP}:\left\{\begin{array}{c}
\left\langle N_{1}(u, v)+M_{1}\left(A_{1}(x), B_{1}(x)\right)-M_{1}\left(A_{1}(y), B_{1}(y)\right), \eta_{1}\left(z_{1}, g_{1}(x)\right)\right\rangle \\
\geq \rho_{1}\left[\phi_{1}\left(g_{1}(x), x\right)-\phi_{1}\left(z_{1}, x\right)\right], \forall z_{1} \in \mathcal{H} \\
\left\langle N_{2}(v, u)+M_{2}\left(A_{2}(y), B_{2}(y)\right)-M_{2}\left(A_{2}(x), B_{2}(x)\right), \eta_{2}\left(z_{2}, g_{2}(y)\right)\right\rangle \\
\geq \rho_{2}\left[\phi_{2}\left(g_{2}(y), y\right)-\phi_{2}\left(z_{2}, y\right)\right], \forall z_{2} \in \mathcal{H} .
\end{array}\right.
$$

Some special cases of GSMVLIP (3) are listed below.

(i) If $M_{1}=M_{2} \equiv 0, g_{1}, g_{2}, P, Q \equiv I$, the identity mappings, then GSMVLIP (3) is equivalent to the problem of finding $\left(x_{1}, x_{2}\right) \in \mathcal{H}_{1} \times \mathcal{H}_{2}$ such that

$$
\left\{\begin{array}{l}
\left\langle M_{1}\left(x_{1}, x_{2}\right), \eta_{1}\left(y_{1}, x_{1}\right)\right\rangle+\phi_{1}\left(x_{1}, y_{1}\right)-\phi_{1}\left(x_{1}, x_{1}\right) \geq 0, \forall y_{1} \in \mathcal{H}_{1}, \\
\left\langle M_{2}\left(x_{1}, x_{2}\right), \eta_{2}\left(y_{2}, x_{2}\right)\right\rangle+\phi_{2}\left(x_{2}, y_{2}\right)-\phi_{2}\left(x_{2}, x_{2}\right) \geq 0, \forall y_{2} \in \mathcal{H}_{2} .
\end{array}\right.
$$

System (4) was studied by Kazmi and Khan [19].

(ii) If $M_{1}=M_{2} \equiv 0, g_{1}=g_{2} \equiv I$, the identity mappings, $\eta_{i}\left(z_{i}, a_{i}\right)=z_{i}-a_{i}, N_{1}(u, v)=S(x, v), N_{2}(v, u)=$ $T(y, u)$ and $\phi_{i}(x, y)=f(x)$, then $G S M V L I P(3)$ coincides with the problem of finding $x, y \in K, u \in E(x)$ and $v \in F(y)$ such that

$$
\left\{\begin{array}{l}
\langle S(x, v)), z-x\rangle+f(z)-f(x) \geq 0, \forall z \in K \\
\langle T(y, u)), z-y\rangle+f(z)-f(y) \geq 0, \forall z \in K
\end{array}\right.
$$

Problem (5) was considered and studied by Li and Li [22].

(iii) If $E=F \equiv I$, the identity mapping, then Problem (5) reduces to the problem of finding $x, y \in K$ such that

$$
\left\{\begin{array}{l}
\langle S(x, y)), z-x\rangle+f(z)-f(x) \geq 0, \forall z \in K \\
\langle T(y, x)), z-y\rangle+f(z)-f(y) \geq 0, \forall z \in K
\end{array}\right.
$$

(iv) If $S(x, y) \equiv s A(y, x)+x-y$ and $T(y, x) \equiv r A(x, y)+y-x$, then System (6) is equivalent to the problem of finding $x, y \in \mathcal{H}$ such that

$$
\left\{\begin{array}{l}
\langle s A(y, x))+x-y, z-x\rangle+\phi(z)-\phi(x) \geq 0, \forall z \in \mathcal{H}, s>0 \\
\langle r A(x, y))+y-x, z-y\rangle+\phi(z)-\phi(y) \geq 0, \forall z \in \mathcal{H}, r>0 .
\end{array}\right.
$$

System (7) was studied by Petrot [27].

(v) If $K$ is a closed convex subsets of $\mathcal{H}$ and $\phi(x)=\delta_{K}(x), \forall x \in K$, where the indicator function $\delta_{K}$ is defined by

$$
\delta_{K}=\left\{\begin{array}{l}
0, \quad \text { if } x \in K \\
+\infty, \text { otherwise }
\end{array}\right.
$$

then the system (7) reduces to the following problem of finding $x, y \in K$ such that

$$
\left\{\begin{array}{l}
\langle s A(y, x))+x-y, z-x\rangle \geq 0, \forall z \in \mathcal{H}, s>0 \\
\langle r A(x, y))+y-x, z-y\rangle \geq 0, \forall z \in \mathcal{H}, r>0
\end{array}\right.
$$

A System of type (8) was studied by Chang et al. [6]. 
In the following theorem, we establish the fixed point formulation of GSMVLIP (3) then we prove the existence of common solution of GSMVLIP (3) for $\alpha \beta$-symmetric $\eta$-monotone mapping and a fixed point problem of nonlinear Lipschitz mappings.

Theorem 2.1. Let $\mathcal{H}$ be a real Hilbert space. For each $i \in\{1,2\} ;$ let $A_{i}, B_{i}, g_{i}: \mathcal{H} \rightarrow \mathcal{H} ; N_{i}: \mathcal{H} \times \mathcal{H} \rightarrow \mathcal{H}$ be the single-valued mappings and $P, Q: \mathcal{H} \rightarrow C B(\mathcal{H})$ be the set-valued mappings. Let $\eta_{i}: \mathcal{H} \times \mathcal{H} \rightarrow \mathcal{H}$ be a $\tau$-Lipschitz continuous mapping such that $\eta_{i}\left(x_{i}, y_{i}\right)+\eta_{i}\left(y_{i}, x_{i}\right)=0$, for all $x_{i}, y_{i} \in \mathcal{H}$. Let $M_{i}: \mathcal{H} \times \mathcal{H} \rightarrow$ $\mathcal{H}$ be an $\alpha_{i} \beta_{i}$-symmetric $\eta_{i}$-monotone mapping with respect to $A_{i}$ and $B_{i}$; let for any $z_{i} \in \mathcal{H}$, the function $\left\langle z_{i}-M_{i}\left(A_{i} x_{i}, B_{i} x_{i}\right), \eta_{i}\left(y_{i}, x_{i}\right\rangle\right.$ be $0-D Q C V$ in $y_{i}$ and let $\phi_{i}: \mathcal{H} \times \mathcal{H} \rightarrow \mathbb{R} \cup\{+\infty\}$ be such that for fixed fixed $x, y \in \mathcal{H}, \phi_{1}\left(g_{1}(\cdot), x\right)$ and $\phi_{2}\left(g_{2}(\cdot), y\right)$ be lower semi-continuous, $\eta$-subdifferential, proper functionals on $\mathcal{H}$ satisfying $g_{1}(\mathcal{H}) \cap \operatorname{dom}\left(\partial_{\eta} \phi\left(g_{1}(\cdot), x\right)\right) \neq \emptyset$ and $g_{2}(\mathcal{H}) \cap \operatorname{dom}\left(\partial_{\eta} \phi\left(g_{2}(\cdot), y\right)\right) \neq \emptyset$. Then $(x, y, u, v)$, where $x, y \in$ $\mathcal{H}, v \in P(x), u \in Q(y)$ is a solution of $G S M V L I P(3)$, if and only if $(x, y, u, v)$ satisfies the following relation:

$$
\begin{aligned}
& x=R_{\left.\rho_{1}, M_{1}(\cdot \cdot) \cdot\right)}^{\partial_{\eta} \phi_{1}\left(g_{1}(\cdot), x\right)}\left[M_{1}\left(A_{1}(y), B_{1}(y)\right)-N_{1}(u, v)\right], \\
& y=R_{\rho_{2}, M_{2}(\cdot, \cdot)}^{\partial_{\eta} \phi_{2}\left(g_{2}(\cdot), y\right)}\left[M_{2}\left(A_{2}(x), B_{2}(x)\right)-N_{2}(v, u)\right],
\end{aligned}
$$

where $\rho_{1}, \rho_{2}>0 \quad$ are constants $\quad$ and $\quad R_{\rho_{1}, M_{1}(\cdot, \cdot)}^{\partial_{\eta} \phi_{1}\left(g_{1}(\cdot), x\right)}=\left[M_{1}\left(A_{1}, B_{1}\right)+\rho \partial_{\eta} \phi\left(g_{1}(\cdot), x\right)\right]^{-1}, R_{\rho_{2}, M_{2}(\cdot, \cdot)}^{\partial_{\eta} \phi_{2}\left(g_{2}(\cdot), y\right)}=$ $\left[M_{2}\left(A_{2}, B_{2}\right)+\rho \partial_{\eta} \phi\left(g_{2}(\cdot), y\right)\right]^{-1}$.

Proof

The conclusion follows directly from the definition of resolvent operators $R_{\rho_{1}, M_{1}(\cdot, \cdot)}^{\partial_{\eta} \phi_{1}\left(g_{1}(\cdot), x\right)}$ and $R_{\rho_{2}, M_{2}(\cdot, \cdot)}^{\partial_{\eta} \phi_{2}\left(g_{2}(\cdot), y\right)}$ of the functionals $\phi_{1}\left(g_{1}(\cdot), x\right)$ and $\phi_{2}\left(g_{2}(\cdot), y\right)$, respectively.

Lemma 2.1. If all the assumptions of the Theorem 2.1 are same and $T: \mathcal{H} \rightarrow \mathcal{H}$ be a nonlinear Lipschitz mapping such that $\{x, y\} \in(G S M V L I P) \cap F(T)$, then

$$
\begin{aligned}
& x=T(x)=T\left[R_{\left.\rho_{1}, M_{1}(\cdot \cdot) \cdot\right)}^{\partial_{\eta} \phi_{1}\left(g_{1}(\cdot), x\right)}\left[M_{1}\left(A_{1}(y), B_{1}(y)\right)-N_{1}(u, v)\right]\right], \\
& y=T(y)=T\left[R_{\rho_{2}, M_{2}(\cdot, \cdot)}^{\left.\partial_{\eta} \phi_{2}(\cdot), y\right)}\left[M_{2}\left(A_{2}(x), B_{2}(x)\right)-N_{2}(v, u)\right]\right] .
\end{aligned}
$$

Based on Lemma 2.1, we suggest the following Mann-type iterative algorithm for finding a common element of the solution set of GSMVLIP (3) involving $\alpha \beta$-symmetric $\eta$-monotone mapping and the set of fixed points $F(T)$ of a nonlinear Lipschitz mapping $T$.

Algorithm 2.1. For any $x_{0}, y_{0} \in \mathcal{H}, v_{0} \in P\left(x_{0}\right), u_{0} \in Q\left(y_{0}\right)$; compute the sequences $\left\{x_{n}\right\},\left\{y_{n}\right\},\left\{u_{n}\right\}$ and $\left\{v_{n}\right\}$ by the following iterative scheme:

$$
\left\{\begin{array}{c}
x_{n+1}=\left(1-\alpha_{n}\right) x_{n}+\alpha_{n} T\left[R_{\rho_{1}, M_{1}(\cdot, \cdot)}^{\partial_{\eta} \phi_{1}\left(g_{1}(\cdot), x_{n}\right)}\left[M_{1}\left(A_{1}\left(y_{n}\right), B_{1}\left(y_{n}\right)\right)-N_{1}\left(u_{n}, v_{n}\right)\right]\right], \\
y_{n}=\left(1-\beta_{n}\right) x_{n}+\beta_{n} T\left[R_{\rho_{2}, M_{2}(\cdot, \cdot)}^{\partial_{\eta} \phi_{2}\left(g_{2}\left(y_{n}\right)\right.}\left[M_{2}\left(A_{2}\left(x_{n}\right), B_{2}\left(x_{n}\right)\right)-N_{2}\left(v_{n}, u_{n}\right)\right]\right],
\end{array}\right.
$$

and

$$
\begin{aligned}
& v_{n} \in P\left(x_{n}\right),\left\|v_{n+1}-v_{n}\right\| \leq \mathcal{D}\left(P\left(x_{n+1}\right), P\left(x_{n}\right)\right) ; \\
& u_{n} \in Q\left(y_{n}\right),\left\|u_{n+1}-u_{n}\right\| \leq \mathcal{D}\left(Q\left(x_{n+1}\right), Q\left(x_{n}\right)\right) ;
\end{aligned}
$$

where $n=1,2,3, \cdots, \rho_{i}>0$ are constants and $\left\{\alpha_{n}\right\},\left\{\beta_{n}\right\}$ are sequences in $[0,1]$.

Theorem 2.2. Let $\mathcal{H}$ be a real Hilbert space. For each $i \in\{1,2\} ;$ let $A_{i}, B_{i}, g_{i}: \mathcal{H} \rightarrow \mathcal{H}$ be the single-valued mappings. Let $M_{i}: \mathcal{H} \times \mathcal{H} \rightarrow \mathcal{H}$ be an $\alpha_{i} \beta_{i}$-symmetric $\eta_{i}$-monotone mapping such that $M_{i}$ be mixed Lipchitz continuous with constant $t_{i}$, mixed strongly monotone with constant $k_{i}$ with respect to $A_{i}$ and $B_{i}$. Let $N_{i}$ : $\mathcal{H} \times \mathcal{H} \rightarrow \mathcal{H}$ be the single-valued mapping such that $N_{i}(\cdot, \cdot)$ be $\gamma_{i}$ and $\gamma_{i}^{\prime}$-Lipschitz continuous in first and second argument, respectively; $P, Q: \mathcal{H} \rightarrow C B(\mathcal{H})$ be the set-valued mappings such that $Q$ is $\mathcal{D}$-Lipchitz continuous with constant $\xi_{1}$, strongly monotone with respect to $N_{1}$ with constant $c_{1}$ and $P$ is $\mathcal{D}$-Lipchitz continuous with 
constant $\xi_{2}$, strongly monotone with respect to $N_{2}$ with constant $c_{2}$. Let $\eta_{i}: \mathcal{H} \times \mathcal{H} \rightarrow \mathcal{H}$ be Lipschitz continuous mapping with constant $\tau_{i}$ such that $\eta_{i}\left(x_{i}, y_{i}\right)=-\eta_{i}\left(y_{i}, x_{i}\right), \forall x_{i}, y_{i} \in \mathcal{H}$, and for any $z \in \mathcal{H}$, the mapping $h_{i}\left(y_{i}, x_{i}\right)=\left\langle z_{i}-M_{i}\left(A_{i} x_{i}, B_{i} x_{i}\right), \eta_{i}\left(z_{i}, g_{i}(\cdot)\right)\right\rangle$ be $0-D Q C V$ in $z_{i}$. Let $\phi_{i}: \mathcal{H} \times \mathcal{H} \rightarrow \mathbb{R} \cup\{+\infty\}$ be such that for each fixed $x, y \in \mathcal{H}, \phi_{1}\left(g_{1}(\cdot), x\right)$ and $\phi_{2}\left(g_{2}(\cdot), y\right)$ be lower semicontinuous, $\eta_{i}$-subdifferentiable, proper functionals on $\mathcal{H}$ satisfying $g_{1}(\mathcal{H}) \cap \operatorname{dom}\left(\partial_{\eta} \phi\left(g_{1}(\cdot), x\right)\right) \neq \emptyset$ and $g_{2}(\mathcal{H}) \cap \operatorname{dom}\left(\partial_{\eta} \phi\left(g_{2}(\cdot), y\right)\right) \neq \emptyset$. Suppose that there exist constants $\rho_{i}>0, \mu_{i}>0$ such that for each $z \in \mathcal{H}$

$$
\begin{aligned}
& \left\|R_{\rho_{1}, M_{1}(\cdot, \cdot)}^{\partial_{\eta} \phi_{1}\left(g_{1}(\cdot), x_{n}\right)}(z)-R_{\rho_{1}, M_{1}(\cdot,)}^{\partial_{\eta} \phi_{1}\left(g_{1}(\cdot), x\right)}(z)\right\| \leq \mu_{1}\left\|x_{n}-x\right\| ; \\
& \left\|R_{\rho_{2}, M_{2}(\cdot, \cdot)}^{\partial_{\eta} \phi_{2}\left(g_{2}(\cdot), y_{n}\right)}(z)-R_{\rho_{2}, M_{2}(\cdot, \cdot)}^{\partial_{\eta} \phi_{\phi}\left(g_{2}(\cdot), y\right)}(z)\right\| \leq \mu_{2}\left\|y_{n}-y\right\| .
\end{aligned}
$$

In addition, GSMVLIP $(3) \cap F(T) \neq \phi$ and the following conditions are satisfied:

(i) $\sum_{n=0}^{\infty} \alpha_{n}=\infty$;

(ii) $\lim _{n \rightarrow \infty} \beta_{n}=1$;

(iii) $k \Omega_{1} \Omega_{3}<\Omega_{3}-k \Omega_{2}$; where,

$\Omega_{1}=\mu_{1}+L_{1} \gamma_{1}^{\prime} \xi_{2}, \Omega_{2}=L_{1}\left(\theta_{1}+\theta_{2}\right)\left[\left(1-\beta_{n}\right)+\beta_{n} k L_{2}\left(\theta_{3}+\theta_{4}\right)\right], \Omega_{3}=\left(1-\beta_{n} k L_{2} \gamma_{2}^{\prime} \xi_{1}-\beta_{n} k \mu_{2}\right)$,

$L_{i}=\frac{\tau_{i}}{\alpha_{i}-\beta_{i}}, \theta_{1}=\sqrt{t_{1}^{2}-2 k_{1}+1}, \theta_{2}=\sqrt{\gamma_{1}^{2} \xi_{1}^{2}-2 c_{1}+1}, \theta_{3}=\sqrt{t_{2}^{2}-2 k_{2}+1}, \sqrt{\gamma_{2}^{2} \xi_{2}^{2}-2 c_{2}+1}$. Then the iterative sequences $\left\{x_{n}\right\},\left\{y_{n}\right\},\left\{u_{n}\right\}$ and $\left\{v_{n}\right\}$ generated by Algorithm 2.1 converges strongly to $x, y, u$ and $v$, respectively and $(x, y, u, v)$ is the common solution of GSMVLIP (3) and $F(T)$.

Proof

By applying Lemma 1.2, Algorithm 2.1, Lipschitz continuity of $T$ and condition (12), we have

$$
\begin{aligned}
& \left\|x_{n+1}-x\right\|=\left\|\left(1-\alpha_{n}\right) x_{n}+\alpha_{n} T\left[R_{\rho_{1}, M_{1}(\cdot, \cdot)}^{\partial_{\eta} \phi_{1}\left(g_{1}(\cdot), x_{n}\right)}\left[M_{1}\left(A_{1}\left(y_{n}\right), B_{1}\left(y_{n}\right)\right)-N_{1}\left(u_{n}, v_{n}\right)\right]\right]-x\right\| \\
& \leq\left(1-\alpha_{n}\right)\left\|x_{n}-x\right\|+\alpha_{n} \| T\left[R_{\rho_{1}, M_{1}(\cdot, \cdot)}^{\partial_{\eta} \phi_{1}\left(g_{1}(\cdot), x_{n}\right)}\left[M_{1}\left(A_{1}\left(y_{n}\right), B_{1}\left(y_{n}\right)\right)-N_{1}\left(u_{n}, v_{n}\right)\right]\right] \\
& -T\left[R_{\rho_{1}, M_{1}(\cdot, \cdot)}^{\partial_{\eta} \phi_{1}\left(g_{1}(\cdot), x\right)}\left[M_{1}\left(A_{1}(y), B_{1}(y)\right)-N_{1}(u, v)\right]\right] \| \\
& \leq\left(1-\alpha_{n}\right)\left\|x_{n}-x\right\|+\alpha_{n} k \| R_{\rho_{1}, M_{1}(\cdot, \cdot)}^{\partial_{\eta} \phi_{1}\left(g_{1}(\cdot), x_{n}\right)}\left[M_{1}\left(A_{1}\left(y_{n}\right), B_{1}\left(y_{n}\right)\right)-N_{1}\left(u_{n}, v_{n}\right)\right] \\
& -R_{\rho_{1}, M_{1}(\cdot, \cdot)}^{\partial_{\eta} \phi_{1}\left(g_{1}(\cdot), x_{n}\right)}\left[M_{1}\left(A_{1}(y), B_{1}(y)\right)-N_{1}(u, v)\right] \| \\
& +\alpha_{n} k \| R_{\rho_{1}, M_{1}(\cdot, \cdot)}^{\partial_{\eta} \phi_{1}\left(g_{1}(\cdot), x_{n}\right)}\left[M_{1}\left(A_{1}(y), B_{1}(y)\right)-N_{1}(u, v)\right] \\
& -R_{\rho_{1}, M_{1}(\cdot, \cdot)}^{\partial_{\eta} \phi_{1}\left(g_{1}(\cdot), x\right)}\left[M_{1}\left(A_{1}(y), B_{1}(y)\right)-N_{1}(u, v)\right] \\
& \leq\left(1-\alpha_{n}\right)\left\|x_{n}-x\right\|+\alpha_{n} k L_{1} \|\left[M_{1}\left(A_{1}\left(y_{n}\right), B_{1}\left(y_{n}\right)\right)-N_{1}\left(u_{n}, v_{n}\right)\right] \\
& -\left[M_{1}\left(A_{1}(y), B_{1}(y)\right)-N_{1}(u, v)\right]\left\|+\alpha_{n} k \mu_{1}\right\| x_{n}-x \| \\
& \leq\left(1-\alpha_{n}\right)\left\|x_{n}-x\right\|+\alpha_{n} k L_{1} \| M_{1}\left(A_{1}\left(y_{n}\right), B_{1}\left(y_{n}\right)\right)-M_{1}\left(A_{1}(y), B_{1}(y)\right) \\
& -\left(y_{n}-y\right)\left\|+\alpha_{n} k L_{1}\right\| N_{1}\left(u_{n}, v_{n}\right)-N_{1}(u, v)-\left(y_{n}-y\right)\left\|+\alpha_{n} k \mu_{1}\right\| x_{n}-x \| \\
& \leq\left(1-\alpha_{n}\right)\left\|x_{n}-x\right\|+\alpha_{n} k L_{1} \| M_{1}\left(A_{1}\left(y_{n}\right), B_{1}\left(y_{n}\right)\right)-M_{1}\left(A_{1}(y), B_{1}(y)\right) \\
& -\left(y_{n}-y\right)\left\|+\alpha_{n} k L_{1}\right\| N_{1}\left(u_{n}, v_{n}\right)-N_{1}\left(u, v_{n}\right)-\left(y_{n}-y\right) \| \\
& +\alpha_{n} k L_{1}\left\|N_{1}\left(u, v_{n}\right)-N_{1}(u, v)\right\|+\alpha_{n} k \mu_{1}\left\|x_{n}-x\right\| .
\end{aligned}
$$

Now,

$$
\begin{aligned}
\| M_{1}\left(A_{1}\left(y_{n}\right), B_{1}\left(y_{n}\right)\right)= & M_{1}\left(A_{1}(y), B_{1}(y)\right)-\left(y_{n}-y\right) \|^{2} \\
= & \left\|M_{1}\left(A_{1}\left(y_{n}\right), B_{1}\left(y_{n}\right)\right)-M_{1}\left(A_{1}(y), B_{1}(y)\right)\right\|^{2} \\
& -2\left\langle M_{1}\left(A_{1}\left(y_{n}\right), B_{1}\left(y_{n}\right)\right)-M_{1}\left(A_{1}(y), B_{1}(y)\right), y_{n}-y\right\rangle+\left\|y_{n}-y\right\|^{2} .
\end{aligned}
$$

Since $M_{1}$ is mixed Lipschitz continuous with constant $t_{1}$ and mixed strongly monotone with constant $k_{1}$ with respect to $A_{1}$ and $B_{1}$, therefore

$$
\begin{aligned}
\left\|M_{1}\left(A_{1}\left(y_{n}\right), B_{1}\left(y_{n}\right)\right)-M_{1}\left(A_{1}(y), B_{1}(y)\right)-\left(y_{n}-y\right)\right\|^{2} & \leq\left(t_{1}^{2}-2 k_{1}+1\right)\left\|y_{n}-y\right\|^{2} \\
& =\theta_{1}^{2}\left\|y_{n}-y\right\|^{2} .
\end{aligned}
$$


Since $Q$ is strongly monotone with respect to $N_{1}$ with constant $c_{1}$ and $N_{1}$ is $\gamma_{1}$-Lipschitz continuous in the first argument and $Q$ is $\mathcal{D}$-Lipschitz continuous with constant $\xi_{1}$, we get

$$
\begin{aligned}
\left\|N_{1}\left(u_{n}, v_{n}\right)-N_{1}\left(u, v_{n}\right)-\left(y_{n}-y\right)\right\|^{2}= & \left\|N_{1}\left(u_{n}, v_{n}\right)-N_{1}\left(u, v_{n}\right)\right\|^{2} \\
& -2\left\langle N_{1}\left(u_{n}, v_{n}\right)-N_{1}\left(u, v_{n}\right), y_{n}-y\right\rangle+\left\|y_{n}-y\right\|^{2} \\
\leq & \left(\gamma_{1}^{2} \xi_{1}^{2}-2 c_{1}+1\right)\left\|y_{n}-y\right\|^{2} \\
= & \theta_{2}^{2}\left\|y_{n}-y\right\|^{2} .
\end{aligned}
$$

Since $N_{1}$ is $\gamma_{1}^{\prime}$-Lipschitz continuous in the second argument and $P$ is $\mathcal{D}$-Lipschitz continuous with constant $\xi_{2}$, we have

$$
\left\|N_{1}\left(u, v_{n}\right)-N_{1}(u, v) \leq \gamma_{1}^{\prime}\right\| v_{n}-v\left\|\leq \gamma_{1}^{\prime} \xi_{2}\right\| x_{n}-x \| .
$$

It follows from (14), (15), (16) and (17) that

$$
\left\|x_{n+1}-x\right\| \leq\left[\left(1-\alpha_{n}\right)+\alpha_{n} k \mu_{1}+\alpha_{n} k L_{1} \gamma_{1}^{\prime} \xi_{2}\right]\left\|x_{n}-x\right\|+\alpha_{n} k L_{1}\left(\theta_{1}+\theta_{2}\right)\left\|y_{n}-y\right\| .
$$

Again employing Lemma 1.2, Algorithm 2.1, Lipschitz continuity of $T$ and condition (13), we have

$$
\begin{aligned}
\left\|y_{n}-y\right\|= & \left\|\left(1-\beta_{n}\right) x_{n}+\beta_{n} T\left[R_{\rho_{2}, M_{2}(\cdot, \cdot)}^{\partial_{\eta} \phi_{2}\left(g_{2}(\cdot), y_{n}\right)}\left[M_{2}\left(A_{2}\left(x_{n}\right), B_{2}\left(x_{n}\right)\right)-N_{2}\left(v_{n}, u_{n}\right)\right]\right]-y\right\| \\
\leq & \left(1-\beta_{n}\right)\left\|x_{n}-y\right\|+\beta_{n} \| T\left[R_{\rho_{2}, M_{2}(\cdot, \cdot)}^{\partial_{\eta} \phi_{2}\left(y_{n}(\cdot),\right.}\left[M_{2}\left(A_{2}\left(x_{n}\right), B_{2}\left(x_{n}\right)\right)-N_{2}\left(v_{n}, u_{n}\right)\right]\right] \\
& -T\left[R_{\rho_{2}, M_{2}(\cdot, \cdot)}^{\partial_{\eta} \phi_{2}\left(g_{2}(\cdot), y\right)}\left[M_{2}\left(A_{2}(x), B_{2}(x)\right)-N_{2}(v, u)\right] \|\right. \\
\leq & \left(1-\beta_{n}\right)\left\|x_{n}-y\right\|+\beta_{n} k \| R_{\rho_{2}, M_{2}(\cdot, \cdot)}^{\partial_{\eta} \phi_{2}\left(g_{2}(\cdot), y_{n}\right)}\left[M_{2}\left(A_{2}\left(x_{n}\right), B_{2}\left(x_{n}\right)\right)-N_{2}\left(v_{n}, u_{n}\right)\right] \\
& -R_{\rho_{2}, M_{2}\left(g_{2}(\cdot, \cdot)\right.}^{\partial_{2} \phi_{2}\left(y_{n}\right)}\left[M_{2}\left(A_{2}(x), B_{2}(x)\right)-N_{2}(v, u)\right] \| \\
& +\beta_{n} k \| R_{\rho_{2}, M_{2}(\cdot, \cdot)}^{\partial_{\eta} \phi_{2}\left(g_{2}(\cdot), y_{n}\right)}\left[M_{2}\left(A_{2}(x), B_{2}(x)\right)-N_{2}(v, u)\right] \\
& -R_{\rho_{2}, M_{2}(\cdot \cdot \cdot)}^{\partial_{\eta} \phi_{2}\left(g_{2}(\cdot), y\right)}\left[M_{2}\left(A_{2}(x), B_{2}(x)\right)-N_{2}(v, u)\right] \| \\
\leq & \left(1-\beta_{n}\right)\left\|x_{n}-x\right\|+\left(1-\beta_{n}\right)\|x-y\| \\
& +\beta_{n} k L_{2} \|\left[M_{2}\left(A_{2}\left(x_{n}\right), B_{2}\left(x_{n}\right)\right)-N_{2}\left(v_{n}, u_{n}\right)\right] \\
& -\left[M_{2}\left(A_{2}(x), B_{2}(x)\right)-N_{2}(v, u)\right]\left\|+\beta_{n} k \mu_{2}\right\| y_{n}-y \| \\
\leq & \left(1-\beta_{n}\right)\left\|x_{n}-x\right\|+\left(1-\beta_{n}\right)\|x-y\|+\beta_{n} k L_{2} \| M_{2}\left(A_{2}\left(x_{n}\right), B_{2}\left(x_{n}\right)\right) \\
& -M_{2}\left(A_{2}(x), B_{2}(x)\right)-\left(x_{n}-x\right) \| \\
& +\beta_{n} k L_{2}\left\|N_{2}\left(v_{n}, u_{n}\right)-N_{2}\left(v, u_{n}\right)-\left(x_{n}-x\right)\right\| \\
& +\beta_{n} k L_{2}\left\|N_{2}\left(v, u_{n}\right)-N_{2}(v, u)\right\|+\beta_{n} k \mu_{2}\left\|y_{n}-y\right\| .
\end{aligned}
$$

Now,

$$
\begin{aligned}
\| M_{2}\left(A_{2}\left(x_{n}\right), B_{2}\left(x_{n}\right)\right)= & M_{2}\left(A_{2}(x), B_{2}(x)\right)-\left(x_{n}-x\right) \|^{2} \\
= & \left\|M_{2}\left(A_{2}\left(x_{n}\right), B_{2}\left(x_{n}\right)\right)-M_{2}\left(A_{2}(x), B_{2}(x)\right)\right\|^{2} \\
& -2\left\langle M_{2}\left(A_{2}\left(x_{n}\right), B_{2}\left(x_{n}\right)\right)-M_{2}\left(A_{2}(x), B_{2}(x)\right), x_{n}-x\right\rangle \\
& +\left\|x_{n}-x\right\|^{2} .
\end{aligned}
$$

Since $M_{2}$ is mixed Lipschitz continuous with constant $t_{2}$ and mixed strongly monotone with constant $k_{2}$ with respect to $A_{2}$ and $B_{2}$, we have

$$
\begin{aligned}
\left\|M_{2}\left(A_{2}\left(x_{n}\right), B_{2}\left(x_{n}\right)\right)-M_{2}\left(A_{2}(x), B_{2}(x)\right)-\left(x_{n}-x\right)\right\|^{2} & \leq\left(t_{2}^{2}-2 k_{2}+1\right)\left\|x_{n}-x\right\|^{2} \\
& =\theta_{3}^{2}\left\|x_{n}-x\right\|^{2}
\end{aligned}
$$

Since $P$ is strongly monotone with respect to $N_{2}$ with constant $c_{2}$ and $N_{2}$ is $\gamma_{2}$-Lipschitz continuous in the first argument and $P$ is $\mathcal{D}$-Lipschitz continuous with constant $\xi_{2}$, we get

$$
\begin{aligned}
\left\|N_{2}\left(v_{n}, u_{n}\right)-N_{2}\left(v, u_{n}\right)-\left(x_{n}-x\right)\right\|^{2}= & \left\|N_{2}\left(v_{n}, u_{n}\right)-N_{2}\left(v, u_{n}\right)\right\|^{2}-2\left\langle N_{2}\left(v_{n}, u_{n}\right)\right. \\
& \left.-N_{2}\left(v, u_{n}\right), x_{n}-x\right\rangle+\left\|x_{n}-x\right\|^{2} \\
\leq & \left(\gamma_{2}^{2} \xi_{2}^{2}-2 c_{2}+1\right)\left\|x_{n}-x\right\|^{2} \\
= & \theta_{4}^{2}\left\|x_{n}-x\right\|^{2} .
\end{aligned}
$$


Since $N_{2}$ is $\gamma_{2}^{\prime}$-Lipschitz continuous in the second argument and $Q$ is $\mathcal{D}$-Lipschitz continuous with constant $\xi_{1}$, we have

$$
\left\|N_{2}\left(v, u_{n}\right)-N_{2}(v, u) \leq \gamma_{2}^{\prime}\right\| u_{n}-u\left\|\leq \gamma_{2}^{\prime} \xi_{1}\right\| y_{n}-y \| .
$$

It follows from (19), (20), (21) and (22) that

$$
\left\|y_{n}-y\right\| \leq\left[\left(1-\beta_{n}\right)+\beta_{n} k L_{2}\left(\theta_{3}+\theta_{4}\right)\right]\left\|x_{n}-x\right\|+\left[\beta_{n} k L_{2} \gamma_{2}^{\prime} \xi_{1}+\beta_{n} k \mu_{2}\right]\left\|y_{n}-y\right\|+\left(1-\beta_{n}\right)\|x-y\|,
$$

which implies that

$$
\left\|y_{n}-y\right\| \leq \frac{\left[\left(1-\beta_{n}\right)+\beta_{n} k L_{2}\left(\theta_{3}+\theta_{4}\right)\right]\left\|x_{n}-x\right\|+\left(1-\beta_{n}\right)\|x-y\|}{\left[1-\beta_{n} k L_{2} \gamma_{2}^{\prime} \xi_{1}-\beta_{n} k \mu_{2}\right]} .
$$

Thus, from (18) and (23), we have

$$
\begin{aligned}
\left\|x_{n+1}-x\right\| \leq & {\left[\left(1-\alpha_{n}\right)+\alpha_{n} k \mu_{1}+\alpha_{n} k L_{1} \gamma_{1}^{\prime} \xi_{2}\right]\left\|x_{n}-x\right\| } \\
& +\alpha_{n} k L_{1}\left(\theta_{1}+\theta_{2}\right)\left[\frac{\left(1-\beta_{n}\right)+\beta_{n} k L_{2}\left(\theta_{3}+\theta_{4}\right)\left\|x_{n}-x\right\|+\left(1-\beta_{n}\right)\|x-y\|}{\left(1-\beta_{n} k L_{2} \gamma_{2}^{\prime} \xi_{1}-\beta_{n} k \mu_{2}\right)}\right] \\
= & {\left[\left(1-\alpha_{n}\right)+\alpha_{n} k \mu_{1}+\alpha_{n} k L_{1} \gamma_{1}^{\prime} \xi_{2}\right]\left\|x_{n}-x\right\| } \\
& +\alpha_{n} k L_{1}\left(\theta_{1}+\theta_{2}\right)\left[\frac{\left(1-\beta_{n}\right)+\beta_{n} k L_{2}\left(\theta_{3}+\theta_{4}\right)}{\left(1-\beta_{n} k L_{2} \gamma_{2}^{\prime} \xi_{1}-\beta_{n} k \mu_{2}\right)}\right]\left\|x_{n}-x\right\| \\
& +\frac{\alpha_{n} k L_{1}\left(\theta_{1}+\theta_{2}\right)\left(1-\beta_{n}\right)}{\left(1-\beta_{n} k L_{2} \gamma_{2}^{\prime} \xi_{1}-\beta_{n} k \mu_{2}\right)}\|x-y\| \\
= & 1-\alpha_{n}\left[1-k\left[\left(\mu_{1}+L_{1} \gamma_{1}^{\prime} \xi_{2}\right)+L_{1}\left(\theta_{1}+\theta_{2}\right) \frac{\left(1-\beta_{n}\right)+\beta_{n} k L_{2}\left(\theta_{3}+\theta_{4}\right)}{\left(1-\beta_{n} k L_{2} \gamma_{2}^{\prime} \xi_{1}-\beta_{n} k \mu_{2}\right)}\right]\right]\left\|x_{n}-x\right\| \\
& +\frac{\alpha_{n} k L_{1}\left(\theta_{1}+\theta_{2}\right)\left(1-\beta_{n}\right)}{\left(1-\beta_{n} k L_{2} \gamma_{2}^{\prime} \xi_{1}-\beta_{n} k \mu_{2}\right)}\|x-y\| \\
= & 1-\alpha_{n}\left[1-k\left(\Omega_{1}+\frac{\Omega_{2}}{\Omega_{3}}\right)\right]+b_{n} .
\end{aligned}
$$

Setting;

$a_{n}=\left\|x_{n}-x\right\|, \lambda_{n}=\alpha_{n}\left[1-k\left(\Omega_{1}+\frac{\Omega_{2}}{\Omega_{3}}\right)\right]$ and $b_{n}=\frac{\alpha_{n} k L_{1}\left(\theta_{1}+\theta_{2}\right)\left(1-\beta_{n}\right)}{\left(1-\beta_{n} k L_{2} \gamma_{2}^{\prime} \xi_{1}-\beta_{n} k \mu_{2}\right)}\|x-y\|$. It follows from condition (iii) that $\lambda_{n} \in(0,1), \forall n \in \mathbb{N}$ and condition (ii) implies that $b_{n}=o\left(\lambda_{n}\right)$. By using condition (iii), we have $\lambda_{n}>\alpha_{n}\left(1-\Omega_{1}\right), \forall n \in \mathbb{N}$. Hence, condition $(i)$ implies that $\sum_{n=0}^{\infty} \lambda_{n}=\infty$. Thus all the conditions of Lemma 1.3 are satisfied and so $\left\|x_{n}-x\right\| \rightarrow 0$ as $n \rightarrow \infty$. Consequently, condition (ii) and (23) implies that $y_{n} \rightarrow y$ as $n \rightarrow \infty$. Since the mappings $P$ and $Q$ are $\mathcal{D}$-Lipschitz continuous and using Algorithm 2.1, it follows that $\left\{u_{n}\right\}$ and $\left\{v_{n}\right\}$ are Cauchy sequences in $\mathcal{H}$ such that $u_{n} \rightarrow u$ and $v_{n} \rightarrow v$ as $n \rightarrow \infty$.

Now, we show that $u \in P(x)$ and $v \in Q(y)$;

$$
\begin{aligned}
d(u, P(x)) & =\inf \{\|u-y\|: y \in P(x)\} \\
& \leq\left\|u-u_{n}\right\|+d\left(u_{n}, P(x)\right) \\
& \leq\left\|u-u_{n}\right\|+\mathcal{D}\left(P\left(x_{n}\right), P(x)\right) \\
& \leq\left\|u-u_{n}\right\|+\xi_{1}\left\|x_{n}-x\right\| \rightarrow 0, \text { as } n \rightarrow \infty,
\end{aligned}
$$

which implies that $d(u, P(x))=0$. Since $P(x) \in \mathcal{C B}(\mathcal{H})$, it follows that $u \in P(x)$. Similarly, we can verify that $v \in Q(y)$. Therefore, in view of Theorem 2.1 and Algorithm 2.1, we conclude that $(x, y, u, v)$ such that $x, y \in \mathcal{H}, u \in P(x)$ and $v \in Q(y)$ is a common solution of GSMVLIP (3) and $F(T)$. 


\section{Convergence Theorem via Altering Points Problem}

In this section, we approximate the solution of GSMVLIP (3) by using the altering points problem. First, we propose a parallel $S$-iterative algorithm for altering points problem associated to generalized system of mixed variational-like inclusion problems involving $\alpha \beta$-symmetric $\eta$-monotone mappings and then we study strong convergence analysis of GSMVLIP (3) by using proposed iterative algorithm.

Let $C$ and $D$ be nonempty closed convex subsets of a real Hilbert space $\mathcal{H}$. Let $S_{1}: C \rightarrow D$ and $S_{2}: D \rightarrow C$ be contraction mappings with Lipschitz constants $\tau_{1}$ and $\tau_{2}$, respectively. Since $S_{2} S_{1}: C \rightarrow C$ is a contraction, there exists a unique element $(x, y) \in C \times D$ of the following altering points problem for operators $S_{1}$ and $S_{2}$.

Find $(x, y) \in C \times D$ such that

$$
\left\{\begin{array}{l}
S_{1}(x)=y \\
S_{2}(y)=x
\end{array}\right.
$$

It is well known that the Picard iterative algorithm converges faster than the Mann iterative algorithm [23] for contraction mappings, see; [2]. Sahu [28] has introduced Normal $S$-iteration Process, whose rate of convergence similar to the Picard iteration process and faster than other fixed point iteration processes (see; [28], Theorem 3.6) which is defined as follows:

$$
\left\{\begin{array}{l}
x_{n+1}=T y_{n}, \\
y_{n}=\left(1-\alpha_{n}\right) x_{n}+\alpha_{n} T\left(x_{n}\right), \forall n \in \mathbb{N},
\end{array}\right.
$$

where $T$ is a self mapping on a convex subset of a normed space $X$ and $\alpha_{n} \subseteq[0,1]$ is a real sequence. Normal $S$-iterative algorithm is independent of Mann algorithm and it has attracted the attention of many researchers, see; for example, $[34,15,30]$ due to fast convergence rate and its simplicity.

Very recently, Gursoy et al. [16] studied the following normal $S$-iterative algorithm:

$$
\left\{\begin{array}{l}
p_{0} \in H, \\
p_{n+1}=S\left[q_{n}-g\left(q_{n}\right)+P_{H}\left[g\left(q_{n}\right)-\sigma T q_{n}\right]\right], \\
q_{n}=\left(1-\xi_{n}\right) p_{n}+\xi_{n} S\left[p_{n}-g\left(p_{n}\right)+P_{H}\left[g\left(p_{n}\right)-\sigma T p_{n}\right]\right],
\end{array}\right.
$$

where $\left\{\xi_{n}\right\}_{n=0}^{\infty} \subset[0,1]$. They have approximated the solution of a generalized nonlinear variational inequalities and studied convergence analysis.

For approximate calculation of altering points of contraction mappings $S_{1}: C \rightarrow D$ and $S_{2}: D \rightarrow C$, motivated by normal $S$-iteration process, Sahu [29] has introduced the following parallel $S$-iteration process:

$$
\left\{\begin{array}{l}
x_{n+1}=T_{2}\left[(1-\alpha) y_{n}+\alpha T_{1} x_{n}\right], \\
y_{n+1}=T_{1}\left[(1-\alpha) x_{n}+\alpha T_{2} y_{n}\right]
\end{array}\right.
$$

where $\alpha \in(0,1)$. Further, Zhao et al. [37] generalized the parallel $S$-iteration process (29) and studied the following parallel $S$-iteration process:

$$
\left\{\begin{array}{l}
x_{n+1}=T_{2}\left[\left(1-\alpha_{n}\right) y_{n}+\alpha_{n} T_{1}\left(x_{n}\right)\right], \\
y_{n+1}=T_{1}\left[\left(1-\beta_{n}\right) x_{n}+\beta_{n} T_{2} y_{n}\right]
\end{array}\right.
$$

where $\left\{\alpha_{n}\right\}$ and $\left\{\beta_{n}\right\}$ are sequences in $(0,1)$. The parallel $S$-iteration process (30) is a natural generalization of the parallel $S$-iteration process (29).

Based on Theorem 2.1 and Definition 1.4, we pose the following altering points problem associated to GSMVLIP (3).

$$
\left\{\begin{array}{l}
S_{1}\left[R_{\rho_{1}, M_{1}(\cdot, \cdot)}^{\partial_{\eta} \phi_{1}\left(g_{1}(\cdot), x\right)}\left[M_{1}\left(A_{1}(y), B_{1}(y)\right)-N_{1}(u, v)\right]\right]=y, \\
S_{2}\left[R_{\rho_{2}, M_{2}(\cdot, \cdot)}^{\partial_{\eta} \phi_{2}\left(g_{2}(\cdot), y\right)}\left[M_{2}\left(A_{2}(x), B_{2}(x)\right)-N_{2}(v, u)\right]\right]=x,
\end{array}\right.
$$


where $S_{1}: C \rightarrow D$ and $S_{2}: D \rightarrow C$ be contraction mappings with Lipschitz constants $\kappa_{1}$ and $\kappa_{2}$, respectively. (3).

Now, we propose following parallel $S$-iterative algorithm to compute the approximate solution of GSMVLIP

Algorithm 3.1. For any $\left(x_{0}, y_{0}\right) \in C \times D$, compute the sequences $\left\{x_{n}, y_{n}\right\} \in C \times D$ generated by the following parallel $S$-iteration scheme.

$$
\left\{\begin{aligned}
x_{n+1} & =S_{2}\left[\left(1-\alpha_{n}\right) R_{\rho_{2}, M_{2}(\cdot, \cdot)}^{\partial_{\eta} \phi_{2}\left(g_{2}(\cdot), y_{n}\right)}\left[M_{2}\left(A_{2}\left(x_{n}\right), B_{2}\left(x_{n}\right)\right)-N_{2}\left(v_{n}, u_{n}\right)\right]\right. \\
& \left.+\alpha_{n} S_{1}\left(R_{\rho_{1}, M_{1}(\cdot, \cdot)}^{\partial_{\eta} \phi_{1}\left(g_{1}(\cdot), x_{n}\right)}\left[M_{1}\left(A_{1}\left(y_{n}\right), B_{1}\left(y_{n}\right)\right)-N_{1}\left(u_{n}, v_{n}\right)\right]\right)\right] \\
y_{n+1} & =S_{1}\left[\left(1-\beta_{n}\right) R_{\rho_{1}, M_{1}(\cdot(\cdot))}^{\left.\partial_{\eta} \phi_{1}(\cdot), x_{n}\right)}\left[M_{1}\left(A_{1}\left(y_{n}\right), B_{1}\left(y_{n}\right)\right)-N_{1}\left(u_{n}, v_{n}\right)\right]\right. \\
& \left.+\beta_{n} S_{2}\left(R_{\rho_{2}, M_{2}(\cdot, \cdot)}^{\partial_{\eta} \phi_{2}\left(g_{2}(\cdot), y_{n}\right)}\left[M_{2}\left(A_{2}\left(x_{n}\right), B_{2}\left(x_{n}\right)\right)-N_{2}\left(v_{n}, u_{n}\right)\right]\right)\right]
\end{aligned}\right.
$$

and

$$
\begin{aligned}
& v_{n} \in P\left(x_{n}\right),\left\|v_{n+1}-v_{n}\right\| \leq \mathcal{D}\left(P\left(x_{n+1}\right), P\left(x_{n}\right)\right) ; \\
& u_{n} \in Q\left(y_{n}\right),\left\|u_{n+1}-u_{n}\right\| \leq \mathcal{D}\left(Q\left(x_{n+1}\right), Q\left(x_{n}\right)\right) ;
\end{aligned}
$$

where $\left\{\alpha_{n}, \beta_{n}\right\}$ is a sequence in $(0,1) \times(0,1)$.

Now we establish strong convergence of the sequences $\left\{x_{n}\right\},\left\{y_{n}\right\},\left\{u_{n}\right\}$ and $\left\{v_{n}\right\}$ generated by parallel $S$ iterative scheme.

Theorem 3.1. Let $C$ and $D$ be nonempty closed convex subsets of a real Hilbert space $\mathcal{H}$. For each $i \in\{1,2\}$; let $A_{i}, B_{i}, g_{i}: \mathcal{H} \rightarrow \mathcal{H}$ be the single-valued mappings. Let $M_{i}: \mathcal{H} \times \mathcal{H} \rightarrow \mathcal{H}$ be $\alpha_{i} \beta_{i}$-symmetric $\eta_{i}$-monotone mappings such that $M_{i}$ be mixed Lipchitz continuous with constant $t_{i}$, mixed strongly monotone with constant $k_{i}$ with respect to $A_{i}$ and $B_{i}$. Let $N_{i}: \mathcal{H} \times \mathcal{H} \rightarrow \mathcal{H}$ be the single-valued mapping such that $N_{i}(\cdot, \cdot)$ be $\gamma_{i}$ and $\gamma_{i}^{\prime}$ Lipschitz continuous in first and second argument, respectively; $P, Q: \mathcal{H} \rightarrow C B(\mathcal{H})$ be the set-valued mappings such that $\overline{D o m(P)} \subseteq D$ and $\overline{D o m(Q)} \subseteq C$ and $Q$ is $\mathcal{D}$-Lipchitz continuous with constant $\xi_{1}$, strongly monotone with respect to $N_{1}$ with constant $c_{1}$ and $P$ is $\mathcal{D}$-Lipchitz continuous with constant $\xi_{2}$, strongly monotone with respect to $N_{2}$ with constant $c_{2}$. Let $S_{1}: C \rightarrow \mathcal{H}$ and $S_{2}: D \rightarrow \mathcal{H}$ be contraction mappings with Lipschitz constant $\kappa_{1}$ and $\kappa_{2}$, respectively. Let $\eta_{i}: \mathcal{H} \times \mathcal{H} \rightarrow \mathcal{H}$ be Lipschitz continuous mapping with constant $\tau_{i}$ such that $\eta_{i}\left(x_{i}, y_{i}\right)=$ $-\eta_{i}\left(y_{i}, x_{i}\right), \forall x_{i}, y_{i} \in \mathcal{H}$, and for any $z \in \mathcal{H}$, the mapping $h_{i}\left(y_{i}, x_{i}\right)=\left\langle z_{i}-M_{i}\left(A_{i} x_{i}, B_{i} x_{i}\right), \eta_{i}\left(z_{i}, g_{i}(\cdot)\right)\right\rangle$ be 0 $D Q C V$ in $z_{i}$. Let $\phi_{i}: \mathcal{H} \times \mathcal{H} \rightarrow \mathbb{R} \cup\{+\infty\}$ be such that for each $x_{i} \in \mathcal{H}, \phi_{1}\left(g_{1}(\cdot), x\right)$ and $\phi_{2}\left(g_{2}(\cdot), y\right)$ be lower semicontinuous, $\eta_{i}$-subdifferentiable, proper functionals. suppose that there exist constants $\rho_{i}>0, \mu_{i}>0$ such that for each $z \in \mathcal{H}$

$$
\begin{aligned}
& \left\|R_{\rho_{1}, M_{1}(\cdot, \cdot)}^{\partial_{\eta} \phi_{1}\left(g_{1}(\cdot), x_{n}\right)}(z)-R_{\rho_{1}, M_{1}(\cdot, \cdot)}^{\partial_{\eta} \phi_{1}\left(g_{1}(\cdot), x\right)}(z)\right\| \leq \mu_{1}\left\|x_{n}-x\right\| . \\
& \left\|R_{\rho_{2}, M_{2}(\cdot, \cdot)}^{\partial_{\eta} \phi_{2}\left(g_{2}(\cdot), y_{n}\right)}(z)-R_{\rho_{2}, M_{2}(\cdot, \cdot)}^{\partial_{\eta} \phi_{2}\left(g_{2}(\cdot), y\right)}(z)\right\| \leq \mu_{2}\left\|y_{n}-y\right\| .
\end{aligned}
$$

In addition, the following condition is satisfied:

$$
\begin{array}{ll}
\Theta=\max \left\{\Theta_{1}, \Theta_{2}\right\} \in[0,1), \\
\text { where, } \quad \Theta_{1}=L_{2}\left(\theta_{3}+\theta_{4}\right)\left(\kappa_{2}+\kappa_{1} \kappa_{2}\right)+\left(L_{1} \gamma_{1}^{\prime} \xi_{2}+\mu_{1}\right)\left(\kappa_{1}+\kappa_{1} \kappa_{2}\right), \\
\Theta_{2}=L_{1}\left(\theta_{1}+\theta_{2}\right)\left(\kappa_{1}+\kappa_{1} \kappa_{2}\right)+\left(L_{2} \gamma_{2}^{\prime} \zeta_{2}+\mu_{2}\right)\left(\kappa_{2}+\kappa_{1} \kappa_{2}\right), \\
\\
\theta_{1}=\sqrt{t_{1}^{2}-2 k_{1}+1}, \theta_{2}=\sqrt{\gamma_{1}^{2} \xi_{1}^{2}-2 c_{1}+1}, \\
& \theta_{3}=\sqrt{t_{2}^{2}-2 k_{2}+1}, \theta_{4}=\sqrt{\gamma_{2}^{2} \zeta_{1}^{2}-2 c_{2}+1}
\end{array}
$$

Then the iterative sequences $\left\{x_{n}\right\},\left\{y_{n}\right\},\left\{u_{n}\right\}$ and $\left\{v_{n}\right\}$ generated by parallel $S$-iterative algorithm 3.1 converges strongly to $x, y, u$ and $v$, respectively. 
Proof

By Algorithm 3.1 and using the Lipschitz continuity of contraction mappings $S_{1}$ and $S_{2}$, we have

$$
\begin{aligned}
& \left\|x_{n+1}-x\right\|=\| S_{2}\left[\left(1-\alpha_{n}\right) R_{\rho_{2}, M_{2}(\cdot, \cdot)}^{\partial_{\eta} \phi_{2}\left(g_{2}(\cdot), y_{n}\right)}\left[M_{2}\left(A_{2}\left(x_{n}\right), B_{2}\left(x_{n}\right)\right)-N_{2}\left(v_{n}, u_{n}\right)\right]\right. \\
& \left.+\alpha_{n} S_{1}\left(R_{\rho_{1}, M_{1}(\cdot, \cdot)}^{\partial_{\eta} \phi_{1}\left(g_{1}(\cdot) x_{n}\right)}\left[M_{1}\left(A_{1}\left(y_{n}\right), B_{1}\left(y_{n}\right)\right)-N_{1}\left(u_{n}, v_{n}\right)\right]\right)\right]-S_{2}(y) \| \\
& \leq \kappa_{2} \|\left(1-\alpha_{n}\right) R_{\rho_{2}, M_{2}(\cdot, \cdot)}^{\partial_{\eta} \phi_{2}\left(g_{2}(\cdot), y_{n}\right)}\left[M_{2}\left(A_{2}\left(x_{n}\right), B_{2}\left(x_{n}\right)\right)-N_{2}\left(v_{n}, u_{n}\right)\right] \\
& +\alpha_{n} S_{1}\left(R_{\rho_{1}, M_{1}(\cdot, \cdot)}^{\partial_{\eta} \phi_{1}\left(g_{1}\left(\cdot, x_{n}\right)\right.}\left[M_{1}\left(A_{1}\left(y_{n}\right), B_{1}\left(y_{n}\right)\right)-N_{1}\left(u_{n}, v_{n}\right)\right]\right)-y \| \\
& \leq \kappa_{2}\left[\left(1-\alpha_{n}\right)\left\|R_{\rho_{2}, M_{2}(\cdot, \cdot)}^{\partial_{\eta} \phi_{2}\left(g_{2}(\cdot), y\right)}\left[M_{2}\left(A_{2}\left(x_{n}\right), B_{2}\left(x_{n}\right)\right)-N_{2}\left(v_{n}, u_{n}\right)\right]-y\right\|\right. \\
& \left.+\alpha_{n}\left\|S_{1}\left(R_{\rho_{1}, M_{1}(\cdot, \cdot)}^{\partial_{\eta} \phi_{1}\left(g_{1}(\cdot), x_{n}\right)}\left[M_{1}\left(A_{1}\left(y_{n}\right), B_{1}\left(y_{n}\right)\right)-N_{1}\left(u_{n}, v_{n}\right)\right]\right)-S_{1}(x)\right\|\right] \\
& \leq \kappa_{2}\left[\left(1-\alpha_{n}\right)\left\|R_{\rho_{2}, M_{2}(\cdot, \cdot)}^{\partial_{\eta} \phi_{2}\left(g_{2}(\cdot), y_{n}\right)}\left[M_{2}\left(A_{2}\left(x_{n}\right), B_{2}\left(x_{n}\right)\right)-N_{2}\left(v_{n}, u_{n}\right)\right]\right\|\right. \\
& -R_{\rho_{2}, M_{2}(\cdot, \cdot)}^{\partial_{\eta} \phi_{2}\left(g_{2}(\cdot), y\right)}\left[M_{2}\left(A_{2}(x), B_{2}(x)\right)-N_{2}(v, u)\right] \| \\
& +\alpha_{n} \kappa_{1} \| R_{\rho_{1}, M_{1}(\cdot, \cdot)}^{\partial_{\eta} \phi_{1}\left(g_{1}(\cdot), x_{n}\right)}\left[M_{1}\left(A_{1}\left(y_{n}\right), B_{1}\left(y_{n}\right)\right)-N_{1}\left(u_{n}, v_{n}\right)\right] \\
& \left.-R_{\rho_{1}, M_{1}(\cdot, \cdot)}^{\partial_{\eta} \phi_{1}\left(g_{1}(\cdot), x\right)}\left[M_{1}\left(A_{1}(y), B_{1}(y)\right)-N_{1}(u, v)\right] \|\right] \\
& \leq \kappa_{2}\left(1-\alpha_{n}\right)\left\|R_{\rho_{2}, M_{2}(\cdot, \cdot)}^{\partial_{\eta} \phi_{2}\left(g_{2}(\cdot), y_{n}\right)}\left[M_{2}\left(A_{2}\left(x_{n}\right), B_{2}\left(x_{n}\right)\right)-N_{2}\left(v_{n}, u_{n}\right)\right]\right\| \\
& -R_{\rho_{2}, M_{2}(\cdot, \cdot)}^{\partial_{\eta} \phi_{2}\left(g_{2}(\cdot), y\right)}\left[M_{2}\left(A_{2}(x), B_{2}(x)\right)-N_{2}(v, u)\right] \| \\
& +\kappa_{2} \alpha_{n} \kappa_{1} \| R_{\rho_{1}, M_{1}(\cdot, \cdot)}^{\partial_{\eta} \phi_{1}\left(g_{1}(\cdot), x_{n}\right)}\left[M_{1}\left(A_{1}\left(y_{n}\right), B_{1}\left(y_{n}\right)\right)-N_{1}\left(u_{n}, v_{n}\right)\right] \\
& -R_{\rho_{1}, M_{1}(\cdot, \cdot)}^{\partial_{\eta} \phi_{1}\left(g_{1}(\cdot), x\right)}\left[M_{1}\left(A_{1}(y), B_{1}(y)\right)-N_{1}(u, v)\right] \| .
\end{aligned}
$$

Following the same arguments as in (14)-(22), we have

$$
\begin{aligned}
\left\|x_{n+1}-x\right\| \leq & \kappa_{2}\left(1-\alpha_{n}\right)\left[L_{2}\left[\left(\theta_{3}+\theta_{4}\right)\left\|x_{n}-x\right\|+\gamma_{2}^{\prime} \xi_{1}\left\|y_{n}-y\right\|\right]+\mu_{2}\left\|y_{n}-y\right\|\right] \\
& +\kappa_{2} \alpha_{n} \kappa_{1}\left[L_{1}\left[\left(\theta_{1}+\theta_{2}\right)\left\|y_{n}-y\right\|+\gamma_{1}^{\prime} \xi_{2}\left\|x_{n}-x\right\|\right]+\mu_{1}\left\|x_{n}-x\right\|\right] .
\end{aligned}
$$

Again using the Lipschitz continuity of contraction mappings $S_{1}$ and $S_{2}$ and by Algorithm 3.1, we have

$$
\begin{aligned}
& \left\|y_{n+1}-y\right\|=\| S_{1}\left[\left(1-\beta_{n}\right) R_{\rho_{1}, M_{1}(\cdot, \cdot)}^{\partial_{\eta} \phi_{1}\left(g_{1}(\cdot), x_{n}\right)}\left[M_{1}\left(A_{1}\left(y_{n}\right), B_{1}\left(y_{n}\right)\right)-N_{1}\left(u_{n}, v_{n}\right)\right]\right. \\
& \left.+\beta_{n} S_{2}\left(R_{\rho_{2}, M_{2}(\cdot, \cdot)}^{\partial_{\eta} \phi_{2}\left(g_{2}(\cdot), y_{n}\right)}\left[M_{2}\left(A_{2}\left(x_{n}\right), B_{2}\left(x_{n}\right)\right)-N_{2}\left(v_{n}, u_{n}\right)\right]\right)\right]-S_{1}(x) \| \\
& \leq \kappa_{1} \|\left(1-\beta_{n}\right) R_{\rho_{1}, M_{1}(\cdot, \cdot)}^{\partial_{\eta} \phi_{1}\left(g_{1}(\cdot), x_{n}\right)}\left[M_{1}\left(A_{1}\left(y_{n}\right), B_{1}\left(y_{n}\right)\right)-N_{1}\left(u_{n}, v_{n}\right)\right] \\
& +\beta_{n} S_{2}\left(R_{\rho_{2}, M_{2}(\cdot, \cdot)}^{\partial_{\eta} \phi_{2}\left(g_{2}\left(\cdot, y_{n}\right)\right.}\left[M_{2}\left(A_{2}\left(x_{n}\right), B_{2}\left(x_{n}\right)\right)-N_{2}\left(v_{n}, u_{n}\right)\right]\right)-x \| \\
& \leq \kappa_{1}\left[\left(1-\beta_{n}\right)\left\|R_{\rho_{12}, M_{1}(\cdot, \cdot)}^{\partial_{\eta} \phi_{1}\left(g_{1}(\cdot), x_{n}\right)}\left[M_{1}\left(A_{1}\left(y_{n}\right), B_{1}\left(y_{n}\right)\right)-N_{1}\left(u_{n}, v_{n}\right)\right]-x\right\|\right. \\
& \left.+\beta_{n}\left\|S_{2}\left(R_{\rho_{2}, M_{2}(\cdot, \cdot)}^{\partial_{\eta} \phi_{2}\left(g_{2}(\cdot), y_{n}\right)}\left[M_{2}\left(A_{2}\left(x_{n}\right), B_{2}\left(x_{n}\right)\right)-N_{2}\left(v_{n}, u_{n}\right)\right]\right)-S_{2}(y)\right\|\right] \\
& \leq \kappa_{1}\left[\left(1-\beta_{n}\right)\left\|R_{\rho_{1}, M_{1}(\cdot, \cdot)}^{\partial_{\eta} \phi_{1}\left(g_{1}(\cdot), x_{n}\right)}\left[M_{1}\left(A_{1}\left(y_{n}\right), B_{1}\left(y_{n}\right)\right)-N_{1}\left(u_{n}, v_{n}\right)\right]\right\|\right. \\
& -R_{\rho_{1}, M_{1}(\cdot, \cdot)}^{\partial_{\eta} \phi_{1}\left(g_{1}(\cdot), y\right)}\left[M_{1}\left(A_{1}(y), B_{1}(y)\right)-N_{1}(u, v)\right] \| \\
& +\beta_{n} \kappa_{2} \| R_{\rho_{2}, M_{2}(\cdot, \cdot)}^{\partial_{\eta} \phi_{2}\left(g_{2}(\cdot), y_{n}\right)}\left[M_{2}\left(A_{2}\left(x_{n}\right), B_{2}\left(x_{n}\right)\right)-N_{2}\left(v_{n}, u_{n}\right)\right] \\
& \left.-R_{\rho_{2}, M_{2}(\cdot, \cdot)}^{\partial_{\eta} \phi_{2}\left(g_{2}(\cdot), y\right)}\left[M_{2}\left(A_{2}(x), B_{2}(x)\right)-N_{2}(v, u)\right] \|\right] \\
& \leq \kappa_{1}\left(1-\beta_{n}\right)\left\|R_{\rho_{1}, M_{1}(\cdot, \cdot)}^{\partial_{1} \phi_{1}\left(g_{1}(\cdot), x_{n}\right)}\left[M_{1}\left(A_{1}\left(y_{n}\right), B_{1}\left(y_{n}\right)\right)-N_{1}\left(u_{n}, v_{n}\right)\right]\right\| \\
& -R_{\rho_{1}, M_{1}(\cdot, \cdot)}^{\partial_{\eta} \phi_{1}\left(g_{1}(\cdot), y\right)}\left[M_{1}\left(A_{1}(y), B_{1}(y)\right)-N_{1}(u, v)\right] \| \\
& +\kappa_{1} \beta_{n} \kappa_{2} \| R_{\rho_{2}, M_{2}(\cdot, \cdot)}^{\partial_{\eta} \phi_{2}\left(g_{2}(\cdot), y_{n}\right)}\left[M_{2}\left(A_{2}\left(x_{n}\right), B_{2}\left(x_{n}\right)\right)-N_{2}\left(v_{n}, u_{n}\right)\right] \\
& \left.-R_{\rho_{2}, M_{2}(\cdot, \cdot)}^{\partial_{\eta} \phi_{2}\left(g_{2}(\cdot), y\right)}\left[M_{2}\left(A_{2}(x), B_{2}(x)\right)-N_{2}(v, u)\right] \|\right] .
\end{aligned}
$$


Again following the same arguments as in (14)-(22), we have

$$
\begin{aligned}
\left\|y_{n+1}-y\right\| \leq & \kappa_{1}\left(1-\beta_{n}\right)\left[L_{1}\left[\left(\theta_{1}+\theta_{2}\right)\left\|y_{n}-y\right\|+\gamma_{1}^{\prime} \xi_{2}\left\|x_{n}-x\right\|\right]+\mu_{1}\left\|x_{n}-x\right\|\right] \\
& +\kappa_{2} \beta_{n} \kappa_{1}\left[L_{2}\left[\left(\theta_{3}+\theta_{4}\right)\left\|x_{n}-x\right\|+\gamma_{2}^{\prime} \xi_{1}\left\|y_{n}-y\right\|\right]+\mu_{2}\left\|y_{n}-y\right\|\right] .
\end{aligned}
$$

Adding the inequalities (37) and (39), we have

$$
\begin{aligned}
\left\|x_{n+1}-x\right\|+\left\|y_{n+1}-y\right\| \leq & \kappa_{2}\left(1-\alpha_{n}\right)\left[L _ { 2 } \left[\left(\theta_{3}+\theta_{4}\right)\left\|x_{n}-x\right\|\right.\right. \\
& \left.\left.+\gamma_{2}^{\prime} \xi_{1}\left\|y_{n}-y\right\|\right]+\mu_{2}\left\|y_{n}-y\right\|\right]+\kappa_{2} \alpha_{n} \kappa_{1}\left[L_{1}\left[\left(\theta_{1}+\theta_{2}\right)\left\|y_{n}-y\right\|\right]\right. \\
& +\mu_{1}\left\|x_{n}-x\right\|+\kappa_{1}\left(1-\beta_{n}\right)\left[L _ { 1 } \left[\left(\theta_{1}+\theta_{2}\right)\left\|y_{n}-y\right\|\right.\right. \\
& \left.\left.+\gamma_{1}^{\prime} \xi_{2}\left\|x_{n}-x\right\|\right]\right]+\mu_{1}\left\|x_{n}-x\right\|+\kappa_{2} \beta_{n} \kappa_{1}\left[L _ { 2 } \left[\left(\theta_{3}+\theta_{4}\right)\left\|x_{n}-x\right\|\right.\right. \\
& \left.\left.+\gamma_{2}^{\prime} \xi_{1}\left\|y_{n}-y\right\|\right]+\mu_{2}\left\|y_{n}-y\right\|\right] \\
\leq & {\left[L_{2}\left(\theta_{3}+\theta_{4}\right)\left(\kappa_{2}+\kappa_{1} \kappa_{2}\right)+\left(\gamma_{1}^{\prime} \xi_{2} L_{1}+\mu_{1}\right)\left(\kappa_{1}+\kappa_{1} \kappa_{2}\right)\right]\left\|x_{n}-x\right\| } \\
& +\left[L_{1}\left(\theta_{1}+\theta_{2}\right)\left(\kappa_{1}+\kappa_{1} \kappa_{2}\right)+\left(\gamma_{2}^{\prime} \xi_{1} L_{2}+\mu_{2}\right)\left(\kappa_{2}+\kappa_{1} \kappa_{2}\right)\right]\left\|y_{n}-y\right\| \\
= & \Theta 1\left\|x_{n}-x\right\|+\Theta_{2}\left\|y_{n}-y\right\| \\
\leq & \Theta\left[\left\|x_{n}-x\right\|+\left\|y_{n}-y\right\|\right],
\end{aligned}
$$

where

$$
\Theta=\max \left\{\Theta_{1}, \Theta_{2}\right\}
$$

and

$$
\left\{\begin{array}{l}
\Theta_{1}=\left[L_{2}\left(\theta_{3}+\theta_{4}\right)\left(\kappa_{2}+\kappa_{1} \kappa_{2}\right)+\left(\gamma_{1}^{\prime} \xi_{2} L_{1}+\mu_{1}\right)\left(\kappa_{1}+\kappa_{1} \kappa_{2}\right)\right] \\
\Theta_{2}=\left[L_{1}\left(\theta_{1}+\theta_{2}\right)\left(\kappa_{1}+\kappa_{1} \kappa_{2}\right)+\left(\gamma_{2}^{\prime} \xi_{1} L_{2}+\mu_{2}\right)\left(\kappa_{2}+\kappa_{1} \kappa_{2}\right)\right] .
\end{array}\right.
$$

It follows from (1) and (40) that

$$
\left\|\left(x_{n+1}, y_{n+1}\right)-(x, y)\right\|_{*} \leq \Theta\left\|\left(x_{n}, y_{n}\right)-(x, y)\right\|_{*} .
$$

Since $\Theta \in(0,1)$ by condition (35), then from Lemma 1.4, we have

$$
\lim _{n \rightarrow \infty}\left\|\left(x_{n}, y_{n}\right)-(x, y)\right\|_{*}=0 .
$$

Thus, we have

$$
\lim _{n \rightarrow \infty}\left\|x_{n}-x\right\|=\lim _{n \rightarrow \infty}\left\|y_{n}-y\right\|=0 .
$$

Therefore, $\left\{x_{n}\right\}$ and $\left\{y_{n}\right\}$ converges to $x$ and $y$, respectively. Since the mappings $P$ and $Q$ are $\mathcal{D}$-Lipschitz continuous and using Algorithm 3.1, it follows that $\left\{u_{n}\right\}$ and $\left\{v_{n}\right\}$ are Cauchy sequences in $\mathcal{H}$ such that $u_{n} \rightarrow u$ and $v_{n} \rightarrow v$ as $n \rightarrow \infty$. It can be proved by using the same techniques as in Theorem 2.2 that $u \in P(x)$ and $v \in Q(y)$. Note that $S_{2} S_{1}: C \rightarrow C$ is a contraction mapping with Lipschitz constant $\kappa_{1} \kappa_{2}$. In view of Theorem 2.1 and (31), we conclude that $(x, y, u, v)$ such that $x, y \in \mathcal{H}, u \in P(x)$ and $v \in Q(y)$ is a unique solution of GSMVLIP (3), where $x$ and $y$ are altering points of mappings $S_{1}$ and $S_{2}$. This completes the proof.

\section{Concluding Remarks}

In this paper, a generalized system of variational-like inclusion problem for $\alpha \beta$-symmetric $\eta$-monotone mapping is considered. We apply Mann type iterative algorithm to analyze the common solution of a generalized system of variational-like inclusion problem for $\alpha \beta$-symmetric $\eta$-monotone mapping and a fixed point problem of nonlinear Lipschitz mapping in Hilbert spaces. Further, we considered altering point problem and proposed a parallel $S$ iterative algorithm. We prove the existence of solution of our system by using altering point problem. The result presented in this paper may be viewed as generalizations and refinements of the results existing in the literature. 


\section{Competing Interests}

The authors declare that there is no competing interests regarding the publication of this article.

\section{Acknowledgement}

This work was supported by the Deanship of Scientific Research (DSR), King Abdulaziz University, Jeddah, under Grant No. (D-023-363-1440). The authors, therefore, gratefully acknowledge the DSR technical and financial support.

\section{REFERENCES}

1. Ahmad, I., Rahaman, M., Ahmad, R.: Relaxed resolvent operator for solving a variational inclusion problem, Statistics, Optimization \& Information Computing, Vol. 4, no. 2, pp. 183-193, (2016)

2. Agrawal, R.P., O’Regan, D., Sahu, D.R.: Fixed Point Theory for Lipschizian-type Mappings with Applications. Springer ScienceBusiness Media, (2009)

3. Ames, W.F.: Numerical Methods for Partial Differential Equations. Academic Press, New York (1992)

4. Aubin, J.P., Ekeland, I.: Applied Nonlinear Analysis. John Wiley and Sons, New York (1984)

5. Baiocchi, C., Capelo, A.: Variational and Quasi-variational Inequalities. J. Wiley and Sons, New York, London (1984)

6. Chang, S. S., Lee, H.W., Chan, C.K.: Generalized system for relaxed cocoercive variational inequalities in Hilbert spaces. Appl. Math. Lett. Vol. 20, pp. 329-334, (2007)

7. Cubiotti, P.: Existence of solutions for lower semicontinuous quasi-equilibrium problems. Comput. Math. Appl. Vol. 30, no. 12, pp. 11-22, (1995)

8. Ding, X.P.: Generalized quasi-variational-like inclusions with nonconvex functionals. Appl. Math. Comput. Vol. 122, no. 3, pp. 267$282,(2001)$

9. Ding, X.P., Lou, C.L.: Perturbed proximal point algorithms for general quasi-variational-like inclusions. J. Comput. Appl. Math. Vol. 113, no. 12, pp. 153-165, (2000)

10. Eckstein, J., Bertsekas, B.P.: On the Douglas-Rachford splitting method and the proximal point algorithm for maximal monotone operators. Math. Program. Vol. 55, no. 1, pp. 293-318, (1992)

11. Fukushima, M.: Equivalent differentiable optimization problems and descent methods for asymmetric variational inequality problems. Math. Program. Vol. 53, pp. 99-110, (1992)

12. Giannessi, F. Maugeri, A.: Variational Inequalities and Network Equilibrium Problems, Plenum Press, New York (1995)

13. Glowinski, R., Lions J.L. Tremolieres, R.: Numerical Analysis of Variational Inequalities, North-Holland, Amsterdam (1981)

14. Glowinski, R.: Numerical Methods for Nonlinear Variational Problems. Springer-Verlag, Berlin (1985)

15. Gursoy, F. A., Khan, R., Ertrk, M., Karakaya, V.: Convergence and data dependency of normal $S$-iterative method for discontinuous operators on Banach space. Numer. Funct. Anal. Optim. Vol. 39, pp. 322-345, (2018)

16. Gursoy, F., Ertrk, M., Abbas, M.: A Picard-type iterative algorithm for general variational inequalities and nonexpansive mappings. Numer. Algorithms https://doi.org/10.1007/s11075-019-00706-w (2019)

17. Hartmann, P., Stampacchia, G.: On some nonlinear elliptic differential functional equations. Acta Math. Vol. 115, no. 1, pp. 271-310, (1966)

18. He, Z.H., Gu, F.: Generalized system for relaxed cocoercive mixed variational inequalities in Hilbert spaces. Appl. Math. Comput. Vol. 214, pp. 26-30, (2009)

19. Kazmi, K. R., Khan, F.A.: Auxiliary problems and algorithm for a system of generalized variational-like inequality problems. Appl. Math. Comput. Vol. 187, pp. 789-796, (2007)

20. Kazmi, K.R., Ahmad, N., Shahzad, M.: Convergence and stability of an iterative algorithm for a system of generalized implicit variational-like inclusions in Banach spaces. Appl. Math. Comput. Vol. 218, pp. 9208-9219, (2012)

21. Lee, C.H., Ansari, Q.H., Yao, J.C.: A perturbed algorithm for strongly nonlinear variational-like inclusions. B. Aust. Math. Soc. Vol. 62, pp. 417-426, (2000)

22. $\mathrm{Li}, \mathrm{X}$. and $\mathrm{Li}, \mathrm{X}$.: A new system of multivalued mixed variational inequality problem. Abstr. Appl. Anal. Volume 2014, Article ID 982606, 7 pages, http://dx.doi.org/10.1155/2014/982606 (2014)

23. Mann, W.R.: Mean value methods in iterations. Proc. Amer. Math. Soc. Vol. 4, pp. 506-510, (1953)

24. Noor, M. A.: General nonlinear variational inequalities. J. Math. Anal. Appl. Vol. 158, pp. 78-84, (1987)

25. Noor, M. A.: General auxiliary principle for variational inequalities. PanAmer. Math. J. Vol. 4, no. 1, pp. 27-44, (1994)

26. Parida, J., Sahoo, M., Kumar, A.: A variational-like inequality problem. B. Aust. Math. Soc. Vol. 39, no. 2, pp. 225-231, (1989)

27. Petrot, N.: A resolvent operator technique for approximate solving of generalized system mixed variational inequality and fixed point problems. Appl. Math. Lett. Vol. 23, pp. 440-445, (2010)

28. Sahu, D.R.: Application of the $S$-iteration process to constrained minimization problem and split feasibility problems. Fixed Point Theory, Vol. 12, pp. 187-204, (2011)

29. Sahu, D.R.: Altering points and applications. Nonlinear Stud. Vol. 21, pp. 349-365, (2014)

30. Sahu, D.R., Yao, J.C., Singh, V. K., Kumar, V.: Semilocal convergence analysis of $S$-iteration process of Newton-Kantorovich like in Banach spaces. J. Optim. Theory. Appl. Vol. 172, pp. 102-127, (2017) 
31. Sahu, N. K., Mahato, N. K., Mohapatra, R. N.: System of nonlinear variational inclusion problems with $(A, \eta)$-maximal monotonicity in Banach space, Statistics, Optimization \& Information Computing, Vol. 5, no. 3, pp. 244-261, (2017)

32. Tian, G.: Generalized quasi-variational-like inequality problem. Math. Oper. Res. Vol. 18, pp. 752-764, (1993)

33. Verma, R. U.: General convergence analysis for two-step projection methods and applications to variational problems. Appl. Math. Lett. Vol. 18, pp. 1286-1292, (2005)

34. Verma, M., Shukla, K.K.: A new accelerated proximal technique for regression with high-dimensional datasets. Knowl. Inf. Syst. Vol. 53, pp. 423-438, (2017)

35. Yao, J.C.: The generalized quasi-variational inequality problem with applications. J. Math. Anal. Appl. Vol. 158, pp. 139-160, (1991)

36. Zhang, S., Guo, X., Luan, D.: Generalized system for relaxed cocoercive mixed variational inequalities and iterative algorithms in Hilbert spaces. An. St. Univ. Ovidius Constanta Vol. 20, no. 3, pp. 131-140, (2012)

37. Zhao, X., Sahu, D.R., Wen, C.F.: Iterative methods for system of variational inclusions involving accretive operators and applications. Fixed Point Theory, Vol. 19, no. 2, pp. 801-822, (2018)

38. Zhou, X.J., Chen, G.: Diagonal convexity conditions for problems in convex analysis and quasi-variational inequalities. J. Math. Anal. Appl. Vol. 132, pp. 213-225, (1988) 